NBER WORKING PAPER SERIES

\title{
BLOCK RECURSIVE EQUILIBRIA FOR STOCHASTIC MODELS OF SEARCH ON THE JOB
}

\author{
Guido Menzio \\ Shouyong Shi \\ Working Paper 14907 \\ http://www.nber.org/papers/w14907
NATIONAL BUREAU OF ECONOMIC RESEARCH
1050 Massachusetts Avenue
Cambridge, MA 02138
April 2009

We are grateful for comments received from participants at the Society for Economic Dynamics Meeting in New Orleans (January 2008) and Cambridge (July 2008), and at the macroeconomics seminars at the University of Michigan, Stanford University and the Minneapolis FED. Discussions with Pete Klenow, Narayana Kocherlakota, Dale Mortensen, Giuseppe Moscarini, and Victor Rios-Rull led to significant improvements in the paper. Menzio gratefully acknowledges the financial support and the hospitality of the Hoover Institution. Shi gratefully acknowledges the financial support from the Bank of Canada Fellowship and from the Social Sciences and Humanities Research Council of Canada. The usual disclaimer applies. The views expressed herein are those of the author(s) and do not necessarily reflect the views of the National Bureau of Economic Research.

NBER working papers are circulated for discussion and comment purposes. They have not been peerreviewed or been subject to the review by the NBER Board of Directors that accompanies official NBER publications.

(C) 2009 by Guido Menzio and Shouyong Shi. All rights reserved. Short sections of text, not to exceed two paragraphs, may be quoted without explicit permission provided that full credit, including $\odot$ notice, is given to the source. 
Block Recursive Equilibria for Stochastic Models of Search on the Job

Guido Menzio and Shouyong Shi

NBER Working Paper No. 14907

April 2009

JEL No. E32,J64

\begin{abstract}
In this paper, we develop a general stochastic model of directed search on the job. Like in the analogous models of random search on the job, the state of the economy in our model includes the infinite-dimensional distribution of workers across different employment states (unemployment, and employment at different wages). Unlike the analogous models of random search on the job, our model admits an equilibrium in which the agents' value and policy functions do not depend on the distribution of workers. We refer to this type of equilibrium as a Block Recursive Equilibrium. Therefore, while solving the equilibrium of a random search model in a stochastic environment is a difficult task both analytically and computationally, solving the Block Recursive Equilibrium of our model is as easy as solving a representative agent model.
\end{abstract}

Guido Menzio

Department of Economics

University of Pennsylvania

467 McNeil Building

3718 Locust Walk

Philadelphia, PA 19104

and NBER

gmenzio@econ.upenn.edu

Shouyong Shi

University of Toronto

Department of Economics

150 St. George Street

Toronto, Ontario

Canada, M5S 3G7

shouyong@chass.utoronto.ca 


\section{Introduction}

This paper studies a general model of search on the job that allows for aggregate shocks, idiosyncratic shocks, and different specifications of the contractual environment. The paper's main result is to formally establish the existence of a type of equilibria, called Block Recursive Equilibria (BRE), which are tracvtable for studying equilibrium dynamics. To attain this result, we depart from the bulk of the literature on search on the job, and we assume that the search process is directed ${ }^{1}$ - in the sense that a worker knows the terms of trade offered by different firms before he chooses where to apply for a job-rather than random (or undirected) - in the sense that a worker does not have any information about terms of trade offered by different firms.

The models of random search on the job by Burdett and Mortensen (1998), Postel-Vinay and Robin (2003), and Burdett and Coles (2003) are a useful tool for studying labor markets because they can simultaneously and parsimoniously explain a number of qualitative features of the data. For example, they can explain the empirical regularities in the transition of workers between the states of employment, unemployment and across jobs that pay different wages (e.g. the negative relationship between job hazard and tenure). They can explain why similar workers employed at similar firms are paid different wages. They can explain why wages tend to increase with tenure and experience. ${ }^{2}$

However, these models are difficult to solve outside of steady-state because the distribution of workers across different employment states (unemployment, and employment at different wages) is an infinite-dimensional state variable which non-trivially affects the agents' value and policy functions. ${ }^{3}$ This technical feature limits the use of these models. For example, a macroeconomist cannot measure the effect of aggregate productivity shocks on the flows of workers across different employment states and on the wage distribution by simply comparing steady-states (unless he has reason to believe that these shocks are very persistent and that the transition phases have negligible length). A public economist cannot measure the welfare effect of a change to the unemployment benefit legislation by comparing two steady-states (unless he has reason to believe

\footnotetext{
${ }^{1}$ The directed search literature was pioneered by Montgomery (1991), Peters (1991), Moen (1997), Acemoglu and Shimer (1999), and Burdett Shi and Wright (2001).

${ }^{2}$ Mortensen (1994), Pissarides (1994), and Barlevy (2002) are other popular models of search on the job. These models have qualitative properties that are very different from those of the models by Burdett and Mortensen (1998), Postel-Vinay and Robin (2002), and Burdett and Coles (2003). For example, they cannot generate residual wage inequality.

${ }^{3}$ Recently, Moscarini and Postel-Vinay (2008) have succeded in characterizing the transitional dynamics of the model by Burdett and Mortensen (1998) (henceforth, BM98). Their results also suggest that it might be possible to solve the equilibrium of BM98 in a fully stochastic environment. The current paper provides a model that has the same qualitative properties as BM98 and can be solved in a stochastic environment as easily as a representative agent model.
} 
that the agents' discount factor is approximately zero and, hence, the transition phases are unimportant). And if an econometrician estimates the steady-state of a model, he has to be careful in using data from a period of time when the fundamentals of the economy have remained approximately unchanged. ${ }^{4}$

Moreover, the hypothesis that the search process is random appears at odds with the existing empirical evidence. For example, in a recent survey of the US labor market, Hall and Krueger (2008, Table 1) find that 84 percent of white, male, non-college workers either "knew exactly" or "had a pretty good idea" about how much their current job would pay from the very beginning of the application process (the time of the first interview). Another piece of evidence against the random search hypothesis and in favor of directed search comes from Holzer, Katz and Krueger (1991). Using data from the 1982 Employment Opportunity Pilot Project Survey, these authors find that firms in high-wage industries tend to attract more applicants per vacancy that firms in industries where the terms of trade are less generous. These findings should not be surprising, as directed search reflects the fundamental idea in economics that prices help a market allocating resources.

In this paper, we consider a stochastic model of directed search on the job. This model is rather general in that it allows for aggregate shocks, idiosyncratic shocks, and for different specifications of the contractual environment (fixed-wage contracts and dynamic contracts). For this model, we prove existence of an equilibrium in which the agents' value and policy functions do not depend on the infinite-dimensional distribution of workers across different employment states. We refer to this equilibrium as a Block Recursive Equilibrium (BRE). Like the equilibrium of the models by Burdett and Mortensen (1998), Postel-Vinay and Robin (2002), and Burdett and Coles (2003), the BRE of our model generates worker flows between employment, unemployment, and across employers; it generates a negative relationship between job hazard and tenure; it generates residual wage inequality, and a positive return to tenure and experience. However, unlike the equilibrium of these other models, the BRE of our model can be easily computed in and out of the steady-state. Therefore, our model can be used, without qualifications, to carry out the labor market measurements that we have described in the previous paragraph.

It is precisely the difference in the nature of the search process that explains why our model

\footnotetext{
${ }^{4}$ Postel-Vinay and Robin (2002) explicitely acknowledge that estimating the steady-state of an OJS model restricts their choice of data: "We have deliberately selected a much shorter period than is available because we want to find out whether it is possible to estimate our model over a homogeneous period of the business cycle. It would have been very hard to defend the assumption of time-invariant parameters (the job offer arrival rate parameters in particular) had we been using a longer panel." Similarly, Jolivet et alii (2006) state that "We choose to restrict our analysis to a 3-year sample for three reasons. [...] Third, the model assumes that the labor market is in a steady-state, an asusmption that would be harder to defend over a longer period of time."
} 
admits a Block Recursive Equilibrium and the models by Burdett and Mortensen (1998), PostelVinay and Robin (2002), and Burdett and Coles (2003) do not. The search process is directed in our model but undirected in the other models. If the search process is directed, workers in different employment states choose to apply for different jobs, because they have different preferences over the probability of getting a job and the value offered by a job. Therefore, the distribution of workers across employment states does not affect the benefit to the firm from creating a vacancy that offers certain terms of trade, the tightness of the labor market and, ultimately, the agents' value and policy functions. In contrast, if the search process is random, workers in different employment states search for the same jobs. Therefore, the distribution of workers across different employment states affects the probability that a firm meets a worker who is willing to accept certain terms of trade, the benefit to the firm of creating a vacancy, the tightness of the labor market and, ultimately, the agents' value and policy functions. At the end of Section 5, we will provide a more detailed explanation for why directed search is important for existence of a BRE.

The main contribution of this paper is to prove existence of a BRE for a relatively general model of directed search on the job which allows for aggregate shocks, idiosyncratic shocks, workers' risk aversion, and for different specifications of the contractual environment. This contribution provides a solid foundation for future applications of models of directed search on the job. Moen and Rosen (2004) were the first to develop a model of search on the job in which the search process is, to some extent, directed (workers can choose where to apply for a job within a restricted set of vacancies). Delacroix and Shi (2006) examine a model of directed search on the job with fixed-wage contracts. However, their analysis only focuses on the steady-state equilibrium. Shi (2008) was the first to prove the existence of a BRE for a model of directed search on the job. However, his model restricts attention to a deterministic environment and to the case of wage/tenure contracts. Menzio and Shi (2008) were the first to prove existence of a BRE for a stochastic model of directed search on the job. In addition, they calibrate their model and use it to measure the contribution of aggregate productivity shocks to the cyclical volatility of unemployment, vacancies, and other labor market variables. However, their model only restricts attention to the case of complete labor contracts. In order to generalize the results from Shi (2008) and Menzio and Shi (2008), the current paper has to develop a different existence proof. Parts of the existence proof in Shi (2008) rely on the absence of aggregate shocks and on the assumption of wage/tenure contracts. The existence proof in Menzio and Shi (2008) is based on the equivalence between the solution to the social planner's problem and the equilibrium allocation. This equivalence does not hold when employment contracts are incomplete. 


\section{The Model}

\subsection{Agents and Markets}

The economy is populated by a continuum of workers with measure one and a continuum of firms with positive measure. Each worker has a periodical utility function $v($.$) defined over$ consumption, where $v: \mathbb{R} \rightarrow \mathbb{R}$ is a twice-continuously differentiable, strictly increasing, weakly concave function such that $v^{\prime}(.) \in\left[\underline{v}^{\prime}, \bar{v}^{\prime}\right], 0<\underline{v}^{\prime} \leq \bar{v}^{\prime}$. Each worker maximizes the expected sum of periodical utilities discounted at the factor $\beta \in(0,1)$. The unemployment benefit is $b$.

Each firm operates a constant returns to scale technology which turns one unit of labor into $y+z$ units of consumption. The first component of productivity, $y$, is common to all firms, and its value lies in the set $Y=\left\{y_{1}, y_{2}, \ldots y_{N(y)}\right\}$, where $\underline{y} \equiv y_{1}<\ldots<y_{N(y)} \equiv \bar{y}$ and $N(y) \geq 2$ is an integer. The second component of productivity, $z$, is specific to each firm-worker pair, and its value lies in the set $Z=\left\{z_{1}, z_{2}, \ldots z_{N(z)}\right\}$, where $\underline{z} \equiv z_{1}<\ldots<z_{N(z)} \equiv \bar{z}$ and $N(z) \geq 1$ is an integer. Each firm maximizes the expected sum of periodical profits discounted at the factor $\beta$.

The labor market is organized in a continuum of submarkets indexed by the expected lifetime utility $x$ that the firms offer to the workers, $x \in X=[\underline{x}, \bar{x}]$, with $\underline{x}<v(b) /(1-\beta)$ and $\bar{x}>$ $v(\bar{y}+\bar{z}) /(1-\beta)$. Specifically, whenever a firm meets a worker in submarket $x$, the firm offers the worker an employment contract that gives him the expected lifetime utility $x$. In submarket $x$, the ratio of the number of vacancies created by firms to the number of workers looking for jobs is given by $\theta(x, \psi) \geq 0$ and is determined in the equilibrium, where $\psi$ is the aggregate state of the economy described below. In the remainder of the paper, we shall refer to $\theta(x, \psi)$ as the tightness of submarket $x .^{5}$

Time is discrete and continues forever. At the beginning of each period, the state of the economy can be summarized by the triple $(y, u, g) \equiv \psi$. The first element of $\psi$ denotes the aggregate component of labor productivity, $y \in Y$. The second element denotes the measure of workers who are unemployed, $u \in[0,1]$. The third element is a function $g: X \times Z \rightarrow[0,1]$, with $g(V, z)$ denoting the measure of workers who are employed at jobs that give them the lifetime utility $\tilde{V} \leq V$ and that have an idiosyncratic component of productivity $\tilde{z} \leq z$.

Each period is divided into four stages: separation, search, matching and production. During the separation stage, an employed worker is forced to move into unemployment with probability $\delta \in(0,1)$. Also, during the separation stage, an employed worker has the option to voluntarily move into unemployment.

\footnotetext{
${ }^{5}$ In submarkets that are not visited by any workers, $\theta(x, \psi)$ is an out-of-equilibrium conjecture that helps determining the equilibrium behavior.
} 
During the second stage, a worker gets the opportunity of searching for a job with a probability that depends on his recent employment history. In particular, if the worker was unemployed at the beginning of the period, he can send an application with probability $\lambda_{u} \in(0,1]$. If the worker was employed at the beginning of the period and did not lose his job during the separation stage, he can search with probability $\lambda_{e} \in(0,1]$. If the worker lost his job during the separation stage, he cannot search immediately. Conditional on being able to search, the worker chooses which submarket to visit. In this sense, search is directed. Also, during the search stage, a firm chooses how many vacancies to create and where to locate them. The cost of maintaining a vacancy for one period is $k>0$. Both workers and firms take the tightness $\theta(x, \psi)$ parametrically. ${ }^{6}$

During the matching stage, the workers and the vacancies in submarket $x$ come together through a frictional meeting process. In particular, a worker meets a vacant job with probability $p(\theta(x, \psi))$, where $p: \mathbb{R}_{+} \rightarrow[0,1]$ is a twice continuously differentiable, strictly increasing, strictly concave function such that $p(0)=0$ and $p^{\prime}(0)<\infty$. Similarly, a vacancy meets a worker with probability $q(\theta(x, \psi))$, where $q: \mathbb{R}_{+} \rightarrow[0,1]$ is a twice continuously differentiable, strictly decreasing function such that $q(\theta)=p(\theta) / \theta, q(0)=1$, and $p\left(q^{-1}().\right)$ being concave. ${ }^{7}$ When a vacancy and a worker meet, the firm that owns the vacancy offers to the worker an employment contract that gives him the lifetime utility $x$. If the worker rejects the offer, he returns to his previous employment position. If the worker accepts the offer, the two parties form a new match. To simplify the exposition, we assume that all new matches have the idiosyncratic component of productivity $z_{0} \in Z$.

During the last stage, an unemployed worker produces and consumes $b \in(0, \bar{y}+\bar{z})$ units of output. A worker employed at a job $z$ produces $y+z$ units of output and consumes $w$ of them, where $w$ is specified by the worker's labor contract. At the end of the production stage, Nature draws next period's aggregate component of productivity, $\hat{y}$, from the probability distribution $\Phi_{\hat{y}}(\hat{y} \mid y)$, and next period's idiosyncratic component of productivity, $\hat{z}$, from the probability distribution $\Phi_{\hat{z}}(\hat{z} \mid z){ }^{8}$ The draws of the idiosyncratic component of productivity are independent across matches. ${ }^{9}$

\footnotetext{
${ }^{6}$ That is, workers and firms treat the tightness $\theta(x, \psi)$ just like households and firms treat prices in a Walrasian Equilibrium.

${ }^{7}$ The last property of $q(\theta)$ is needed to guarantee that the worker's search problem is strictly concave and its solution unique. The reader should notice that this property (as well as the other properties of $p$ and $q$ ) are satisfied by many standard specifications of the matching process. For example, it is satisfied by the CES matching process $q(\theta)=\left[\alpha /\left(\alpha+\theta^{\sigma}\right)\right]^{1 / \sigma}, p(\theta)=\theta\left[\alpha /\left(\alpha+\theta^{\sigma}\right)\right]^{1 / \sigma}, \alpha \in(0,1)$ and $1 \geq \sigma>0$.

${ }^{8}$ Throughout this paper, the caret on a variable indicates the variable in the next period.

${ }^{9}$ In order to keep the exposition simple, we have chosen to restrict attention to aggregate and idiosyncratic shocks that affect only labor productivity. However, the proof of the existence of a Block Recursive Equilibrium does not depend on this choice, and can be easily generalized to the case in which aggregate and idiosyncratic shocks affect the search process, the value of unemployment, labor income taxes, etc.
} 


\subsection{Contractual Environment}

We consider two alternative contractual environments. In the first environment, the firm commits to an employment contract that specifies the worker's wage as a function of the history of realizations of the idiosyncratic productivity of the match, $z$, the history of realizations of the aggregate state of the economy, $\psi$, and the history of realizations of a two-point lottery that is drawn at the beginning of every production stage. ${ }^{10}$ In the remainder of the paper, we shall refer to this as the "dynamic contract" environment, since we will formulate the contracts recursively as in the literature on dynamic contracts (e.g., Atkeson and Lucas, 1992). ${ }^{11}$ In the second environment, the firm commits to a wage that remains constant throughout the entire duration of the employment relationship. This constant wage is allowed to depend only on the outcome of a two-point lottery that is drawn at the beginning of the employment relationship. In the remainder of the paper, we shall refer to this as the "fixed-wage contract" environment.

We are interested in these two contractual environments because they have been the focus of the literature on random search on the job. The "dynamic contract" environment generalizes the environment considered by Burdett and Coles (2003) and Shi (2008) to an economy with stochastic productivity. ${ }^{12}$ The "fixed-wage contract" environment is the same environment that has been considered by Burdett and Mortensen (1998), van den Berg and Ridder (1998), and Jolivet et al. (2006). Notice that, under both specifications of the environment, contracts are incomplete because wages cannot be made contingent upon the outside offers received by the worker.

\subsection{Worker's Problem}

Consider a worker whose current employment position gives him a lifetime utility $V$ and who has the opportunity to look for a job at the beginning of the search stage. His search decision is to choose which submarket $x$ to visit. If the worker visits submarket $x$, he succeeds in finding a job with probability $p(\theta(x, \psi))$, and fails with probability $1-p(\theta(x, \psi))$. If he succeeds, he enters the production stage in a new employment relationship which gives him the lifetime utility $x$. If he fails to find a new match (or if he does not apply for a job), he enters the production stage by

\footnotetext{
${ }^{10} \mathrm{We}$ allow the specifications of the employment contract to depend on the history of realization of these twopoint lotteries in order to guarantee that the firm's value function is concave. In this sense, lotteries play a similar role in our model as in Prescott and Townsend (1984).

${ }^{11}$ In contrast to most models in the literature on dynamic contracts, however, there is no private information in our model, and a worker can quit for another job or into unemployment in any period during the contract.

${ }^{12}$ In the special case where workers are risk neutral, the dynamic contracts considered in this paper attain the same allocation as the complete contracts considered in Menzio and Shi (2008) do. Therefore, the proof of the existence of a Block Recursive Equilibrium in this paper generalizes the existence proof in Menzio and Shi (2008).
} 
retaining his current employment position, which gives him a lifetime utility $V$. Therefore, the worker's lifetime utility at the beginning of the search stage is $V+\max \{0, R(V, \psi)\}$, where

$$
R(V, \psi)=\max _{x \in X} p(\theta(x, \psi))(x-V)
$$

Denote $m(V, \psi)$ as the solution to the maximization problem in $(2.1)$, and $\tilde{p}(V, \psi)$ as the composite function $p(\theta(m(V, \psi), \psi))$.

Next, consider an unemployed worker at the beginning of the production stage, and denote as $U(\psi)$ his lifetime utility. In the current period, the worker produces and consumes $b$ units of output. During the next search stage period, the worker is unemployed and has the opportunity to look for a job with probability $\lambda_{u}$. Therefore, the worker's lifetime utility $U(\psi)$ is equal to

$$
U(\psi)=v(b)+\beta \mathbb{E}_{\hat{\psi}}\left[U(\hat{\psi})+\lambda_{u} \max \{0, R(U(\hat{\psi}), \hat{\psi})\}\right] .
$$

\subsection{Firm's Problem}

\subsubsection{Dynamic Contracts}

Consider a firm that has just met a worker in submarket $x$. The firm offers to the worker an employment contract that specifies his wage at every future date as a function of the realized history of the idiosyncratic productivity of the match, the realized history of the aggregate state of the economy, and the history of realizations of a two-point lottery that is drawn at the beginning of every production stage. The firm chooses the employment contract in order to maximize its profits and provide the worker with the promised lifetime utility $x$. Charcaterizing the solution to this problem is difficult because the dimension of the history upon which wages are contingent grows to infinity with time. However, following the literature on dynamic contracts (e.g. Atkeson and Lucas, 1992), we can rewrite this problem recursively by using the worker's lifetime utility as an auxiliary state variable. ${ }^{13}$

In the recursive formulation of the problem, the state of the contract at the beginning of each production stage is described by the worker's lifetime utility, $V$, by the state of the aggregate economy, $\psi$, and by the idiosyncratic productivity of the match, $z$. Let $s$ denote $(\psi, z)$. Given $V$ and $s$, the firm chooses a two-point lottery over the worker's wage $w$ in the current period, the worker's probability $d$ of becoming unemployed in the next separation stage, and the worker's

\footnotetext{
${ }^{13}$ More precisely, we can prove that the value function of the firm's contracting problem is the unique solution to the recursive problem (2.3). Also, we can prove that the firm's contracting problem yields the same solutions as the recurive problem (2.3). The proof of these equivalence results is standard. However, all details are available upon request.
} 
lifetime utility $\hat{V}$ at the beginning of the next production stage. That is, the firm chooses a twopoint lottery $c=\left(\pi_{i}, w_{i}, d_{i}, \hat{V}_{i}\right)_{i=1}^{2}$, where $\pi_{i}$ is the probability with which the realization of the lottery is $\left(w_{i}, d_{i}, \hat{V}_{i}\right)$. The firm's choice is subject to a promise-keeping constraint, which requires $c$ to provide the worker with the lifetime utility $V$, and to an individual rationality constraint, which requires the separation probability $d$ to be consistent with the worker's incentives to quit. The firm chooses $c$ to maximize the sum of its profits in the current period and its profits from the next period onward. Therefore, the firm's maximized value $J(V, s)$ is equal to

$$
\begin{aligned}
J(V, s) & =\max _{\pi_{i}, w_{i}, \hat{d}_{i}, \hat{V}_{i}} \sum_{i=1}^{2} \pi_{i}\left\{y+z-w_{i}+\beta \mathbb{E}_{\hat{s}}\left[\left(1-d_{i}(\hat{s})\right)\left(1-\lambda_{e} \tilde{p}\left(\hat{V}_{i}(\hat{s}), \hat{\psi}\right)\right) J\left(\hat{V}_{i}(\hat{s}), \hat{s}\right)\right]\right\} \\
\text { s.t. } & \pi_{i} \in[0,1], w_{i} \in \mathbb{R}, d_{i}: \Psi \times Z \rightarrow[\delta, 1], \hat{V}_{i}: \Psi \times Z \rightarrow X, \quad \text { for } i=1,2, \\
& \sum_{i=1}^{2} \pi_{i}=1, \quad d_{i}(\hat{s})=\left\{\delta \text { if } U(\hat{\psi}) \leq \hat{V}_{i}(\hat{s})+\lambda_{e} R\left(\hat{V}_{i}(\hat{s}), \hat{\psi}\right), \quad 1 \text { else }\right\}, \\
& \sum_{i=1}^{2} \pi_{i}\left\{v\left(w_{i}\right)+\beta \mathbb{E}_{\hat{s}}\left[d_{i}(\hat{s}) U(\hat{\psi})+\left(1-d_{i}(\hat{s})\right)\left(\hat{V}_{i}(\hat{s})+\lambda_{e} R\left(\hat{V}_{i}(\hat{s}), \hat{\psi}\right)\right)\right]\right\}=V .
\end{aligned}
$$

We denote the optimal policy function associated with $(2.3)$ as $c(V, s)=\left(\pi_{i}, w_{i}, d_{i}, \hat{V}_{i}\right)_{i=1}^{2}$, where $\pi_{i}=\pi_{i}(V, s), w_{i}=w_{i}(V, s), d_{i}=d_{i}(V, s, \hat{s})$, and $\hat{V}_{i}=\hat{V}_{i}(V, s, \hat{s})$, for $i=1,2$.

\subsubsection{Fixed-Wage Contracts}

With fixed-wage contracts, we assume that workers are risk averse; i.e., $v(w)=w$ for all $w$. Consider a worker who is employed for a wage of $w$ at the beginning of the production stage, and denote as $H(w, \psi)$ his lifetime utility. In the current period, the worker consumes $w$ units of output. During the next separation stage, the worker is forced to become unemployed with probability $\delta$, and has the option of keeping his job with probability $1-\delta$. If the worker becomes unemployed, he does not have the opportunity to look for a new job during the next search stage. If the workers keeps his job, he has the opportunity to look for a better job with probability $\lambda_{e}$. Therefore, the worker's lifetime utility $H(w, \psi)$ is equal to

$$
\begin{aligned}
& H(w, \psi)=w+\beta \mathbb{E}_{\hat{\psi}}\left\{d(\hat{\psi}) U(\hat{\psi})+(1-d(\hat{\psi}))\left[H(w, \hat{\psi})+\lambda_{e} \max \{0, R(H(w, \hat{\psi}), \hat{\psi})\}\right]\right\} \\
& d(\hat{\psi})=\left\{\delta \text { if } U(\hat{\psi}) \leq H(w, \hat{\psi})+\lambda_{e} \max \{0, R(H(w, \hat{\psi}), \hat{\psi})\}, \quad 1 \text { else }\right\} .
\end{aligned}
$$

We denote as $h(V, \psi)$ the wage that provides an employed worker with the lifetime utility $V$. That is, $h(V, \psi)$ is the solution of the equation $H(w, \psi)=V$ with respect to $w$.

Next, consider a firm that employs a worker for a wage of $w$ at the beginning of the production stage, and denote as $K(w, s)$ its lifetime profit. In the current period, the firm's profit is given by $y+z-w$. The discounted sum of profits from the next period onward is $(1-d(\hat{\psi}))(1-$ 
$\left.\lambda_{e} \tilde{p}(H(w, \hat{\psi}), \hat{\psi})\right) K(w, \hat{s})$. Therefore, $K(w, s)$ is equal to

$$
\begin{aligned}
& \left.K(w, s)=y+z-w+\beta \mathbb{E}_{\hat{s}}\left[(1-d(\hat{\psi}))\left(1-\lambda_{e} \tilde{p}(H(w, \hat{\psi}), \hat{\psi})\right) K(w, \hat{s})\right)\right] \\
& d(\hat{\psi})=\left\{\delta \text { if } U(\hat{\psi}) \leq H(w, \hat{\psi})+\lambda_{e} \max \{0, R(H(w, \hat{\psi}), \hat{\psi})\}, \quad 1 \text { else }\right\} .
\end{aligned}
$$

Finally, consider a firm that has just met a worker in submarket $x=V$, and denote as $J\left(V, \psi, z_{0}\right)$ its lifetime profit. The firm offers to the worker a two-point lottery over the constant wage $w$. The firm's offer is required to provide the worker with the lifetime utility $V$ (if accepted). Therefore, the firm's lifetime profit $J\left(V, \psi, z_{0}\right)$ is equal to

$$
\begin{array}{cl}
J\left(V, \psi, z_{0}\right) & =\max _{\pi_{i}, \tilde{V}_{i}} \sum_{i=1}^{2} \pi_{i} K\left(h\left(\tilde{V}_{i}, \psi\right), \psi, z_{0}\right), \\
\text { s.t. } & \pi_{i} \in[0,1], \tilde{V}_{i} \in X, \quad \text { for } i=1,2, \\
& \sum_{i=1}^{2} \pi_{i}=1, \quad \sum_{i=1}^{2} \pi_{i} \tilde{V}_{i}=V .
\end{array}
$$

We denote the optimal policy function associated with $(2.6)$ as $c=\left(\pi_{i}, \tilde{V}_{i}\right)_{i=1}^{2}$, where $\pi_{i}=\pi_{i}(V, s)$ and $\tilde{V}_{i}=\tilde{V}_{i}(V, s)$, for $i=1,2$.

\subsection{Market Tightness}

During the search stage, a firm chooses how many vacancies to create and where to locate them. The firm's benefit of creating a vacancy in submarket $x$ is the product between the probability of meeting a worker, $q(\theta(x, \psi))$, and the value of meeting a worker, $J\left(x, \psi, z_{0}\right)$. The firm's cost of creating a vacancy in submarket $x$ is $k$. When the benefit is strictly smaller than the cost, the firm's optimal policy is to create no vacancies in $x$. When the benefit is strictly greater than the cost, the firm's optimal policy is to create infinitely many vacancies in $x$. And when the benefit and the cost are equal, the firm's profi is independent of the number of vacancies it creates in submarket $x$.

In any submarket that is visited by a positive number of workers, the tightness $\theta(x, \psi)$ is consistent with the firm's optimal creation strategy if and only if

$$
k \geq q(\theta(x, \psi)) J\left(x, \psi, z_{0}\right)
$$

and $\theta(x, \psi) \geq 0$, with complementary slackness. In any submarket that workers do not visit, the tightness $\theta(x, \psi)$ is consistent with the firm's optimal creation strategy if and only if $q(\theta(x, \psi)) J\left(x, \psi, z_{0}\right)$ is smaller than or equal to $k$. Following most of the literature on directed search, we restrict attention to equilibria in which the tightness $\theta(x, \psi)$ satisfies condition $(2.7)$ in all submarkets. ${ }^{14}$

\footnotetext{
${ }^{14}$ See Acemoglu and Shimer (1999), Delacroix and Shi (2006), Menzio (2007), and Shi (2008)
} 


\section{Block Recursive Equilibrium: Definition and Procedure}

The previous section motivates the following definition of a recursive equilibrium:

Definition 3.1. A Recursive Equilibrium consists of a market tightness function $\theta: X \times \Psi \rightarrow \mathbb{R}_{+}$, a search value function $R: X \times \Psi \rightarrow \mathbb{R}$, a policy function $m: X \times \Psi \rightarrow X$, an unemployment value function $U: \Psi \rightarrow \mathbb{R}$, a firm's value function $J: X \times \Psi \times Z \rightarrow \mathbb{R}$, a contract policy function $c: X \times \Psi \times Z \rightarrow C$, and a transition probability function for the aggregate state of the economy $\Phi_{\hat{\psi}}: \Psi \times \Psi \rightarrow[0,1]$. These functions satisfy the following requirements:

(i) $\theta$ satisfies (2.7) for all $(x, \psi) \in X \times \Psi$;

(ii) $R$ satisfies (2.1) for all $(V, \psi) \in X \times \Psi$, and $m$ is the associated policy function;

(iii) $U$ satisfies (2.2) for all $\psi \in \Psi$;

(iv) $J$ satisfies (2.3) or (2.6) for all $(V, \psi, z) \in X \times \Psi \times Z$, and $c$ is the associated policy function;

(v) $\Phi_{\hat{\psi}}$ is derived from the policy functions, $(m, c)$, and the probability distributions for $(\hat{y}, \hat{z})$.

Solving a recursive equilibrium outside of the steady-state requires solving a system of functional equations in which the unknown functions depend on the entire distribution of workers across employment states, $(u, g)$. Since this distribution is a large dimensional object (for example, it is an infinite dimensional object in the version of the model with dynamic contracts), solving a recursive equilibrium outside of the steady-state is a difficult task both analytically and computationally. In contrast, solving the following class of equilibria is much easier because it involves solving a system of functional equations in which the unknown functions have at most three dimensions.

Definition 3.2. A Block Recursive Equilibrium (BRE) is a recursive equilibrium such that the functions $\{\theta, R, m, U, J, c\}$ depend on the aggregate state of the economy, $\psi$, only through the aggregate component of productivity, $y$, and not through the distribution of workers across employment states, $(u, g)$.

In this paper, we establish existence of a Block Recursive Equilibrium. To this aim, we define $\mathcal{J}(X \times Y \times Z)$ (henceforth $\mathcal{J})$ as the set of firm's value functions $J: X \times Y \times Z \rightarrow \mathbb{R}$ such that: (J1) For all $(y, z) \in Y \times Z$ and all $V_{1}, V_{2} \in X$, with $V_{1} \leq V_{2}$, the difference $J\left(V_{2}, y, z\right)-J\left(V_{1}, y, z\right)$ is bounded between $-\bar{B}_{J}\left(V_{2}-V_{1}\right)$ and $-\underline{B}_{J}\left(V_{2}-V_{1}\right)$, where $\bar{B}_{J} \geq \underline{B}_{J}>0$ are some constants; (J2) For all $(V, y, z) \in X \times Y \times Z, J(V, y, z)$ is bounded in $[\underline{J}, \bar{J}] ;{ }^{15}$ (J3) For all $(y, z) \in Y \times Z$,

\footnotetext{
${ }^{15}$ We list this property separately in addition to (J1) to emphasize the fact that the bounds $\underline{J}$ and $\bar{J}$ are uniform for all functions in the set $J$ and for all $(V, y, z)$.
} 
$J(V, y, z)$ is concave in $V$. In words, a firm's value function $J$ in the set $\mathcal{J}$ depends on $\psi$ only through $y$. Moreover, a firm's value function $J$ in the set $\mathcal{J}$ is bounded; it is strictly decreasing and weakly concave in $V$; and its "derivative" with respect to $V$ is bounded above and below, i.e. $J$ is bi-Lipschitz continuous in $V .{ }^{16}$ In Appendix A, we prove that $\mathcal{J}$ is a non-empty, bounded, closed and convex subset of the space of bounded, continuous functions on $X \times Y \times Z$, with the sup norm. ${ }^{17}$

In Section 4, we take an arbitrary firm's value function $J$ from the set $\mathcal{J}$. Given $J$, we prove that the market tightness function, $\theta$, that solves the equilibrium condition (2.7) depends on the state of the economy, $\psi$, only through the aggregate component of productivity, $y$, and not through the distribution of workers across employment states, $(u, g)$. Intuitively, since the value of filling a vacancy in submarket $x$ does not depend on the distribution of workers and the cost of creating a vacancy is constant, the equilibrium probability of filling a vacancy in submarket $x$, and hence the tightness of submarket $x$, must be independent of the distribution of workers.

Given $\theta$, we prove that the search value function, $R$, that solves the equilibrium condition (2.1) depends on $\psi$ only through $y$. Intuitively, $R$ does not depend on $(u, g)$, because neither the probability that a worker finds a job in submarket $x$ nor the benefit to a worker from finding a job in submarket $x$ depends on the employment status of other workers in the economy. Given $R$, we prove that the unemployment value function, $U$, that solves the equilibrium condition (2.2) depends on $\psi$ only through $y$. Intuitively, $U$ does not depend on $(u, g)$, because neither the output of an unemployed worker nor his return to searching depends on the distribution of workers across different employment states.

In Section 5, we insert $J, \theta, R$, and $U$ in the RHS of the equilibrium condition (2.3) to construct an update of the firm's value function, where $T$ maps the function $J$ with which the above procedure starts into a new function. First, we prove that $T J$ depends on $\psi$ only through $y$. Intuitively, $T J$ does not depend on $(u, g)$ because the output of a match in the current period, the probability that a match survives until the next production stage, and the value to the firm of a match at the next production stage are all independent of the distribution of workers across employment states. Second, we prove that $T J$ is bounded between $\underline{J}$ and $\bar{J}$; it is strictly decreasing and weakly concave in $V$; and its "derivative" with respect to $V$ is bounded between $-\bar{B}_{J}$ and

\footnotetext{
${ }^{16}$ A function $J(x)$ is Lipschitz over $x \in X$ if $\left|J\left(x_{2}\right)-J\left(x_{1}\right)\right| \leq B_{1}\left|x_{2}-x_{1}\right|$ for all $x_{1}, x_{2} \in X$, where $B_{1}$ is a finite constant. The function is bi-Lipschitz if, in addition, $\left|J\left(x_{2}\right)-J\left(x_{1}\right)\right| \geq B_{2}\left|x_{2}-x_{1}\right|$ for all $x_{1}, x_{2} \in X$, where $B_{2}$ is a strictly positive constant. We need the firm's value function $J$ to be bi-Lipschitz in order to ensure the set $\mathcal{J}$ to be closed and convex. In addition, bi-Lipschitz continuity implies that $J$ is strictly decreasing, a property that will be used to establish important properties such as those of the market tightness.

${ }^{17}$ Throughout this paper, the norm is the sup norm unless it is specified otherwise.
} 
$-\underline{B}_{J}$. Intuitively, the firm's updated value function, $T J$, is bounded because the output of the match is bounded and there is time discounting; $T J$ is decreasing because a firm finds it costly to provide a worker with higher lifetime utility; $T J$ is concave because the contract between a firm and a worker includes a lottery; and the "derivative" of $T J$ is bounded because the derivative of the worker's utility function is bounded. Third, we prove that $T J$ is continuous in $J$.

From the first two properties of $T J$ above, it follows that the equilibrium operator $T$ maps the set of firm's value functions $\mathcal{J}$ into itself. From the third property of $T J$, it follows that the equilibrium operator $T$ is continuous in $J$. From bi-Lipschitz continuity of $T J$, it follows that the family of functions $T(\mathcal{J})$ is equicontinuous. Overall, the equilibrium operator $T$ satisfies the assumptions of Schauder's fixed point theorem (see Stokey and Lucas with Prescott, 1989), and, hence, there exists a $J^{*} \in \mathcal{J}$ such that $J^{*}=T J^{*}$. Applying one more time the above procedure that leads to the mapping $T$, but with the firm's value function $J^{*}$, we can construct equilibrium policy functions $\theta^{*}, R^{*}, m^{*}, U^{*}$, and $c^{*}$. These functions and $J^{*}$ constitute a Block Recursive Equilibrium for the version of the model with dynamic contracts. In Section 6, we use a similar argument to prove existence of a Block Recursive Equilibrium for the version of the model with fixed-wage contracts.

\section{General Properties of an Equilibrium}

\subsection{Market Tightness}

Start with an arbitrary value function of the firm, $J \in \mathcal{J}$, we construct the market tightness function and analyze its properties. For all $(x, \psi) \in X \times \Psi$ such that $J\left(x, y, z_{0}\right) \geq k$, the solution to the equilibrium condition (2.7) is given by a market tightness of $q^{-1}\left(k / J\left(x, y, z_{0}\right)\right)$, where $q^{-1}\left(k / J\left(x, y, z_{0}\right)\right)$ is bounded between 0 and $\bar{\theta} \equiv q^{-1}(k / \bar{J})$. For all $(x, \psi) \in X \times \Psi$ such that $J\left(x, y, z_{0}\right)<k$, the solution to the equilibrium condition (2.7) is given by a market tightness of 0 . The condition $J\left(x, y, z_{0}\right) \geq k$ is satisfied if and only if $x \leq \tilde{x}(y)$, where $\tilde{x}(y)$ is the solution to the equation $J\left(x, y, z_{0}\right)<k$ with respect to $x$. From these observations, it follows that the function $\theta: X \times Y \rightarrow[0, \bar{\theta}]$ defined as

$$
\theta(x, y)= \begin{cases}q^{-1}\left(k / J\left(x, y, z_{0}\right)\right), & \text { if } x \leq \tilde{x}(y), \\ 0, & \text { else }\end{cases}
$$

is the unique solution to the equilibrium condition (2.7) for all $(x, \psi) \in X \times \Psi$.

The market tightness function, $\theta$, has several properties. First, $\theta$ depends on the aggregate state of the economy, $\psi$, only through the aggregate component of productivity, $y$, and not through the distribution of workers across different employment states, $(u, g)$. Second, the market 
tightness function, $\theta$, is strictly decreasing with respect to $x$. Intuitively, since the firm's value from filling a vacancy is lower in a submarket with a higher $x$, the firm's probability of filling a vacancy must be higher. Third, the market tightness function, $\theta$, is Lipschitz continuous in $x$ for all $x$, and bi-Lipschitz in $x$ for $x<\tilde{x}(y)$. Intuitively, since the firm's value function, $J$, is bi-Lipschitz continuous in $x$ and the derivative of the function $q^{-1}($.$) is bounded, the market$ tightness function defined in (4.1) is also bi-Lipschitz continuous for all such $x$ that $\theta(x, y)>0$. Finally, the probability that a worker meets a vacancy in submarket $x, p(\theta(x, y))$, decreases at an increasing rate as $x$ increases. This property follows from the concavity of the firm's value function $J$ and of the composite function $p\left(q^{-1}().\right)$. These properties of $\theta$ are summarized in the following lemma and proved in Appendix B.

Lemma 4.1. (i) For all $y \in Y$, the market tightness function, $\theta$, is such that

$$
\begin{array}{ll}
\frac{\bar{B}_{J}}{q^{\prime}(\bar{\theta}) k}\left(x_{2}-x_{1}\right) \leq \theta\left(x_{2}, y\right)-\theta\left(x_{1}, y\right) \leq \frac{\underline{B}_{J} k}{q^{\prime}(0) \bar{J}^{2}}\left(x_{2}-x_{1}\right), & \text { if } x_{1} \leq x_{2} \leq \tilde{x}(y), \\
\frac{\bar{B}_{J}}{q^{\prime}(\bar{\theta}) k}\left(x_{2}-x_{1}\right) \leq \theta\left(x_{2}, y\right)-\theta\left(x_{1}, y\right) \leq 0, & \text { if } x_{1} \leq \tilde{x}(y) \leq x_{2}, \\
\theta\left(x_{2}, y\right)-\theta\left(x_{1}, y\right)=0, & \text { if } \tilde{x}(y) \leq x_{1} \leq x_{2},
\end{array}
$$

where $\underline{B}_{J}$ and $\bar{B}_{J}$ are the bi-Lipschitz bounds on all functions in $\mathcal{J}$. (ii) For all $y \in Y$ and all $x \in[\underline{x}, \tilde{x}(y)]$, the composite function $p(\theta(x, y))$ is strictly decreasing and strictly concave in $x$.

The function $\theta(x, y)$ constructed above depends on the arbitrary function $J$. Consider two arbitrary functions $J_{n}, J_{r} \in \mathcal{J}$. Let $\theta_{n}$ denote the market tightness function computed with $J_{n}$, and $\theta_{r}$ with $J_{r}$. In the following lemma, we prove that, if the distance between $J_{n}$ and $J_{r}$ converges to zero, so does the distance between $\theta_{n}$ and $\theta_{r}$. That is, we prove that the market tightness function, $\theta$, is continuous with respect to the firm's value function $J$ with which it is computed. This result will be used in Sections 5 and 6 to establish that the equilibrium operator $T$ is continuous.

Lemma 4.2. For any $\rho>0$ and any $J_{n}, J_{r} \in \mathcal{J}$, if $\left\|J_{n}-J_{r}\right\|<\rho$, then

$$
\left\|\theta_{n}-\theta_{r}\right\|<\alpha_{\theta} \rho, \quad \alpha_{\theta} \equiv-\bar{B}_{J} /\left[q^{\prime}(\bar{\theta}) \underline{B}_{J} k\right] .
$$

ProOF: For the sake of brevity, let us suppress the dependence of various functions on $(y, z)$. Let $\rho>0$ be an arbitrary real number. Let $J_{n}$ and $J_{r}$ be arbitrary functions in $\mathcal{J}$ such that $\left\|J_{n}-J_{r}\right\|<\rho$. Let $y$ be an arbitrary point in $Y$. From property (J1) of the set $\mathcal{J}$, it follows 
that $J_{r}\left(x+\underline{B}_{J}^{-1} \rho\right)-J_{r}(x) \leq-\rho$ and, hence, $J_{r}(x)-\rho \geq J_{r}\left(x+\underline{B}_{J}^{-1} \rho\right)$. From property (J1), it also follows that $J_{r}(x)-J_{r}\left(x-\underline{B}_{J}^{-1} \rho\right) \leq-\rho$ and, hence, $J_{r}(x)+\rho \leq J_{r}\left(x-\underline{B}_{J}^{-1} \rho\right)$. From these observations and $\left\|J_{n}-J_{r}\right\|<\rho$, it follows that

$$
\begin{aligned}
& J_{n}(x)<J_{r}(x)+\rho \leq J_{r}\left(x-\underline{B}_{J}^{-1} \rho\right), \\
& J_{n}(x)>J_{r}(x)-\rho \geq J_{r}\left(x+\underline{B}_{J}^{-1} \rho\right) .
\end{aligned}
$$

From the first line in (4.4) and equation (4.1), it follows that $\theta_{n}(x) \leq \theta_{r}\left(x-\underline{B}_{J}^{-1} \rho\right)$. Similarly, from the second line in (4.4) and equation (4.1), it follows that $\theta_{n}(x) \geq \theta_{r}\left(x+\underline{B}_{J}^{-1} \rho\right)$. Hence,

$$
\begin{aligned}
& \theta_{n}(x)-\theta_{r}(x)<\theta_{r}\left(x-\underline{B}_{J}^{-1} \rho\right)-\theta_{r}(x) \leq \alpha_{\theta} \rho, \\
& \theta_{n}(x)-\theta_{r}(x)>\theta_{r}\left(x+\underline{B}_{J}^{-1} \rho\right)-\theta_{r}(x) \geq-\alpha_{\theta} \rho,
\end{aligned}
$$

where $\alpha_{\theta}$ is defined in (4.3). Thus, $\left|\theta_{n}(x)-\theta_{r}(x)\right| \leq \alpha_{\theta} \rho$. Since this result holds for all $(x, y, z) \in$ $X \times Y \times Z$, we conclude that $\left\|\theta_{n}-\theta_{r}\right\|<\alpha_{\theta} \rho$.

\subsection{Search Problem}

Given the firm's value function $J \in \mathcal{J}$, the market tightness function $\theta$ defined in (4.1) satisfies the equilibrium condition (2.7). Given $\theta$, the search value function, $R$, that satisfies the equilibrium condition (2.1) is equal to $\max _{x \in X} f(x, V, y)$ for all $(x, \psi) \in X \times \Psi$, where $f(x, V, y) \equiv p(\theta(x, y))(x-V)$. Note that, for all $(V, \psi) \in X \times \Psi$, the objective function, $f$, depends on the aggregate state of the economy, $\psi$, through the aggregate component of productivity, $y$, and not through the distribution of workers across different employment states, $(u, g)$. Also, note that the choice set, $X$, is independent of the aggregate state of the economy, $\psi$. From these observations, it follows that the optimal search decision and the search value function, $R$, depend on $\psi$ only through $y$ and not through $(u, g)$.

Given $\theta$, a search policy function satisfies the equilibrium condition (2.1) if its value belongs to $\arg \max _{x \in X} f(x, V, y)$ for all $(V, \psi) \in X \times \Psi$. For all $(V, \psi) \in X \times \Psi$, the objective function, $f$, is negative for all $x$ in the interval $[\underline{x}, V]$, strictly positive for all $x$ in the interval $(V, \tilde{x}(y))$, and equal to zero for all $x$ in the interval $[\tilde{x}(y), \bar{x}]$. Moreover, the objective function is strictly concave in $x$ for all $x$ in the interval $(V, \tilde{x}(y))$ (Shi, 2008, Lemma 3.1). Therefore, if $V<\tilde{x}(y)$, the argmax is unique and belongs to the interval $(V, \tilde{x}(y))$. If $V \geq \tilde{x}(y)$, the argmax includes any point between $V$ and $\bar{x}$. From these observations, it follows that the function $m: X \times Y \rightarrow X$ defined as

$$
m(V, y)= \begin{cases}\arg \max _{x \in X} f(x, V, y), & \text { if } V<x(y) \\ V, & \text { else }\end{cases}
$$


is the unique solution to the equilibrium condition (2.1).

In Lemma 4.3, we prove that the search value function, $R$, is decreasing in $V$. Intuitively, since the value to a worker from finding a job in submarket $x$ is decreasing in the value of his current employment position, $V$, and the probability that a worker finds a job in submarket $x$ is independent of $V$, the return to search is decreasing in $V$. Also, in Lemma 4.3, we prove that the search policy function, $m$, is increasing in $V$. Intuitively, since the marginal rate of substitution between the value offered by a new job and the probability of finding a new job is decreasing in $V$, the optimal search strategy is increasing in $V$. Monotonicity of $m$ in $V$ implies that the workers choose to separate themselves into different submarkets in job search. Moreover, in Lemmas 4.3 and 4.3, we prove that the search value and policy functions are Lipschitz continuous in $V$. These results will be used in Sections 5 and 6 to prove that the equilibrium operator $T$ is continuous.

Lemma 4.3. For all $y \in Y$ and all $V_{1}, V_{2} \in X, V_{1} \leq V_{2}$, the search value function, $R$, is such that

$$
-\left(V_{2}-V_{1}\right) \leq R\left(V_{2}, y\right)-R\left(V_{1}, y\right) \leq 0
$$

and the search policy function, $m$, is such that

$$
0 \leq m\left(V_{2}, y\right)-m\left(V_{1}, y\right) \leq V_{2}-V_{1}
$$

Proof: For the sake of brevity, let us suppress the dependence of the functions $\theta, \tilde{x}, m$ and $p$ on $y$. Let $V_{1}$ and $V_{2}$ be two arbitrary points in $X$, with $V_{1} \leq V_{2}$. The difference $R\left(V_{2}\right)-R\left(V_{1}\right)$ is such that

$$
\begin{aligned}
& R\left(V_{2}\right)-R\left(V_{1}\right) \leq f\left(m\left(V_{2}\right), V_{2}\right)-f\left(m\left(V_{2}\right), V_{1}\right) \leq-p\left(\theta\left(m\left(V_{2}\right)\right)\right)\left(V_{2}-V_{1}\right) \leq 0, \\
& R\left(V_{2}\right)-R\left(V_{1}\right) \geq f\left(m\left(V_{1}\right), V_{2}\right)-f\left(m\left(V_{1}\right), V_{1}\right) \geq-p\left(\theta\left(m\left(V_{1}\right)\right)\right)\left(V_{2}-V_{1}\right) \geq-\left(V_{2}-V_{1}\right) .
\end{aligned}
$$

where the first inequality in both lines makes use of the fact that $R\left(V_{i}\right)$ is equal to $f\left(m\left(V_{i}\right), V_{i}\right)$ and greater than $f\left(m\left(V_{-i}\right), V_{i}\right)$ where $-i \neq i$ and $i,-i=1,2$. Thus, (4.6) holds.

Turn to (4.7). If $V_{1} \geq \tilde{x}$, then $m\left(V_{2}\right)=V_{2}$ and $m\left(V_{1}\right)=V_{1}$. In this case, (4.7) clearly holds. If $V_{2} \geq \tilde{x} \geq V_{1}$, then $m\left(V_{2}\right)=V_{2}$ and $m\left(V_{1}\right) \in\left(V_{1}, \tilde{x}\right)$. Also in this case, (4.7) holds.

Now, consider the remaining case where $V_{1} \leq V_{2}<\tilde{x}$. Since $f\left(m\left(V_{1}\right), V_{1}\right) \geq f\left(m\left(V_{2}\right), V_{1}\right)$ and $f\left(m\left(V_{2}\right), V_{2}\right) \geq f\left(m\left(V_{1}\right), V_{2}\right)$, we have

$$
\begin{aligned}
0 & \geq f\left(m\left(V_{2}\right), V_{1}\right)-f\left(m\left(V_{1}\right), V_{1}\right)+f\left(m\left(V_{1}\right), V_{2}\right)-f\left(m\left(V_{2}\right), V_{2}\right) \\
& =p\left(\theta\left(m\left(V_{2}\right)\right)\right)\left(V_{2}-V_{1}\right)-p\left(\theta\left(m\left(V_{1}\right)\right)\right)\left(V_{2}-V_{1}\right) \\
& =\left[p\left(\theta\left(m\left(V_{2}\right)\right)\right)-p\left(\theta\left(m\left(V_{1}\right)\right)\right)\right]\left(V_{2}-V_{1}\right) .
\end{aligned}
$$


Since $p(\theta(x))$ is decreasing in $x$, the previous inequality implies that $m\left(V_{2}\right)-m\left(V_{1}\right) \geq 0$.

If $m\left(V_{2}\right)-m\left(V_{1}\right)=0,(4.7)$ holds. If $m\left(V_{2}\right)-m\left(V_{1}\right)>0$, let $\Delta$ be an arbitrary real number in the open interval between 0 and $\left(m\left(V_{2}\right)-m\left(V_{1}\right)\right) / 2$. Using the definition of $R$, we can deduce from the inequality $f\left(m\left(V_{1}\right), V_{1}\right) \geq f\left(m\left(V_{1}\right)+\Delta, V_{1}\right)$ the following result:

$$
m\left(V_{1}\right)-V_{1} \geq \frac{p\left(\theta\left(m\left(V_{1}\right)+\Delta\right)\right) \Delta}{p\left(\theta\left(m\left(V_{1}\right)\right)\right)-p\left(\theta\left(m\left(V_{1}\right)+\Delta\right)\right)} .
$$

Similarly, because $f\left(m\left(V_{2}\right), V_{2}\right) \geq f\left(m\left(V_{2}\right)-\Delta, V_{2}\right)$, we have

$$
m\left(V_{2}\right)-V_{2} \leq \frac{p\left(\theta\left(m\left(V_{2}\right)-\Delta\right)\right) \Delta}{p\left(\theta\left(m\left(V_{2}\right)-\Delta\right)\right)-p\left(\theta\left(m\left(V_{2}\right)\right)\right)} .
$$

Recall that the function $p(\theta(x))$ is decreasing and concave in $x$ for all $x \leq \tilde{x}(y)$. Since $m\left(V_{1}\right)+$ $\Delta \leq m\left(V_{2}\right)-\Delta$, then $p\left(\theta\left(m\left(V_{1}\right)+\Delta\right)\right) \geq p\left(\theta\left(m\left(V_{2}\right)-\Delta\right)\right)$. Similarly, since $m\left(V_{1}\right)<m\left(V_{2}\right)$, $p\left(\theta\left(m\left(V_{1}\right)\right)\right)-p\left(\theta\left(m\left(V_{1}\right)+\Delta\right)\right) \leq p\left(\theta\left(m\left(V_{2}\right)-\Delta\right)\right)-p\left(\theta\left(m\left(V_{2}\right)\right)\right)$. From these observations and the inequalities above, it follows that $m\left(V_{2}\right)-m\left(V_{1}\right) \leq V_{2}-V_{1}$. Hence, (4.7) holds.

Now we turn to the composite function $\tilde{p}(V, y)=p(\theta(m(V, y), y))$. The probability $\tilde{p}(V, y)$ is the probability that an employed worker finds a new job during the matching stage, given that his current job gives him the lifetime utility $V$ and the aggregate component of productivity is $y$. The following corollary states that the function $\tilde{p}(V, y)$ is decreasing and Lipschitz continuous in $V:$

Corollary 4.4. For all $y \in Y$ and all $V_{1}, V_{2} \in X, V_{1} \leq V_{2}$, the quitting probability $\tilde{p}$ is such that

$$
-\bar{B}_{p}\left(V_{2}-V_{1}\right) \leq \tilde{p}\left(V_{2}, y\right)-\tilde{p}\left(V_{1}, y\right) \leq-\underline{B}_{p}\left(V_{2}-V_{1}\right)
$$

where $\bar{B}_{p}=-p^{\prime}(0) \bar{B}_{J} /\left[q^{\prime}(\bar{\theta}) k\right]>0$ and $\underline{B}_{p}=0$.

Proof: Let $y$ be an arbitrary point in $Y$, and let $V_{1}, V_{2}$ be two points in $X$ with $V_{1} \leq V_{2}$. From Lemma 4.3, it follows that the difference $m\left(V_{2}, y\right)-m\left(V_{1}, y\right)$ is greater than 0 and smaller than $V_{2}-V_{1}$. From Lemma 4.1, it follows that the difference $\theta\left(m\left(V_{2}, y\right), y\right)-\theta\left(m\left(V_{2}, y\right), y\right)$ is greater than $\left(V_{2}-V_{1}\right) \bar{B}_{J} /\left[q^{\prime}(\bar{\theta}) k\right]$ and smaller than 0 . Finally, since $p$ is a concave function of $\theta$, the difference $p\left(\theta\left(m\left(V_{2}, y\right), y\right)\right)-p\left(\theta\left(m\left(V_{2}, y\right), y\right)\right)$ is such that

$$
p^{\prime}(0) \bar{B}_{J} /\left[q^{\prime}(\bar{\theta}) k\right]\left(V_{2}-V_{1}\right) \leq p\left(\theta\left(m\left(V_{2}, y\right), y\right)\right)-p\left(\theta\left(m\left(V_{1}, y\right), y\right)\right) \leq 0 .
$$

These are the bounds in (4.8).

Now, consider two arbitrary functions $J_{n}, J_{r} \in \mathcal{J}$. Let $\theta_{n}$ denote the market tightness function computed with $J_{n}, R_{n}$ and $m_{n}$ the search value and policy functions computed with 
$\theta_{n}$, and $\tilde{p}_{n}(V, y)$ the composite function $p\left(\theta_{n}\left(m_{n}(V, y), y\right)\right)$. Similarly, let $\theta_{r}$ denote the market tightness function computed with $J_{r}, R_{r}$ and $m_{r}$ the search value and policy functions computed with $\theta_{r}$, and $\tilde{p}_{r}(V, y)$ the composite function $p\left(\theta_{r}\left(m_{r}(V, y), y\right)\right)$. In the following lemma, we prove that, if the distance between $J_{n}$ and $J_{r}$ converges to zero, so does the distance between $R_{n}$ and $R_{r}$ and the distance between $\tilde{p}_{n}$ and $\tilde{p}_{r}$. That is, we prove that the search value function $R$ and the separation probability $\tilde{p}$ are continuous with respect to the firm's value function $J$. These results will be used in Sections 5 and 6 to establish that the equilibrium operator $T$ is continuous.

Lemma 4.5. For any $\rho>0$ and any $J_{n}, J_{r} \in \mathcal{J}$, if $\left\|J_{n}-J_{r}\right\|<\rho$, then

$$
\begin{gathered}
\left\|R_{n}-R_{r}\right\|<\alpha_{R} \rho, \quad \alpha_{R} \equiv p^{\prime}(0) \alpha_{\theta}(\bar{x}-\underline{x}), \\
\left\|\tilde{p}_{n}-\tilde{p}_{r}\right\|<\alpha_{p}(\rho), \quad \alpha_{p}(\rho) \equiv \max \left\{2 \bar{B}_{p} \rho^{1 / 2}+p^{\prime}(0) \alpha_{\theta} \rho, 2 \alpha_{R} \rho^{1 / 2}\right\} .
\end{gathered}
$$

As $\rho \rightarrow 0, \alpha_{p}(\rho) \rightarrow 0$.

ProOF: For the sake of brevity, let us suppress the dependence of various functions on $V$ and $y$. Let $\rho>0$ be an arbitrary real number. Let $J_{n}$ and $J_{r}$ be arbitrary functions in $\mathcal{J}$ such that $\left\|J_{n}-J_{r}\right\|<\rho$. Let $(V, y)$ be an arbitrary point in $X \times Y$. The distance between $R_{n}(V, y)$ and $R_{r}(V, y)$ is such that

$$
\begin{aligned}
\left|R_{n}-R_{r}\right| & \leq \max _{x \in X}\left|\left[p\left(\theta_{n}(x)\right)-p\left(\theta_{r}(x)\right)\right](x-V)\right| \\
& \leq\left\{\max _{x \in X}\left|p\left(\theta_{n}(x)\right)-p\left(\theta_{r}(x)\right)\right|\right\}\left\{\max _{x \in X}|x-V|\right\} \\
& \leq\left\{\max _{x \in X}\left|\int_{\theta_{r}(x)}^{\theta_{n}(x)} p^{\prime}(t) d t\right|\right\}(\bar{x}-\underline{x})<p^{\prime}(0) \alpha_{\theta}(\bar{x}-\underline{x}) \rho,
\end{aligned}
$$

where the last inequality makes use of the bounds in (4.3). Since this result holds for all $(V, y) \in$ $X \times Y$, we conclude that $\left\|R_{n}-R_{r}\right\|<\alpha_{R} \rho$.

Now, consider the function $\tilde{p}$. Without loss of generality, assume $m_{r}(V, y) \leq m_{n}(V, y)$. (If $m_{r}(V, y)>m_{n}(V, y)$, just switch the roles of $m_{n}$ and $m_{r}$ in the proof below.) First, consider the case where $p\left(\theta_{r}\left(m_{r}\right)\right) \leq p\left(\theta_{n}\left(m_{n}\right)\right)$. In this case, the distance between $p\left(\theta_{n}\left(m_{n}\right)\right)$ and $p\left(\theta_{r}\left(m_{r}\right)\right)$ is such that

$$
(0 \leq) p\left(\theta_{n}\left(m_{n}\right)\right)-p\left(\theta_{r}\left(m_{r}\right)\right) \leq p\left(\theta_{n}\left(m_{r}\right)\right)-p\left(\theta_{r}\left(m_{r}\right)\right)<p^{\prime}(0) \alpha_{\theta} \rho,
$$

where the first inequality makes use of the fact that $p\left(\theta_{n}(x)\right)$ is decreasing in $x$ and $m_{n} \geq m_{r}$, and the second inequality makes use of the bounds in (4.3). 
Second, consider the case where $p\left(\theta_{r}\left(m_{r}\right)\right)>p\left(\theta_{n}\left(m_{n}\right)\right)$ and $m_{n}-2 \rho^{1 / 2} \leq m_{r} \leq m_{n}$. In this case, the distance between $p\left(\theta_{n}\left(m_{n}\right)\right)$ and $p\left(\theta_{r}\left(m_{r}\right)\right)$ is such that

$$
\begin{aligned}
& (0<) p\left(\theta_{r}\left(m_{r}\right)\right)-p\left(\theta_{n}\left(m_{n}\right)\right) \\
& =p\left(\theta_{r}\left(m_{r}\right)\right)-p\left(\theta_{r}\left(m_{n}\right)\right)+p\left(\theta_{r}\left(m_{n}\right)\right)-p\left(\theta_{n}\left(m_{n}\right)\right) \\
& <2 \bar{B}_{p} \rho^{1 / 2}+p^{\prime}(0) \alpha_{\theta} \rho,
\end{aligned}
$$

where the last inequality makes use of the bounds in (4.8) and in (4.3). Note that this bound is larger than the one in the previous case.

Finally, consider the remaining case where $p\left(\theta_{r}\left(m_{r}\right)\right)>p\left(\theta_{n}\left(m_{n}\right)\right)$ and $m_{r}<m_{n}-2 \rho^{1 / 2}<m_{n}$. First, note that $m_{r} \geq V$, because $m_{r} \in\left(V, \tilde{x}_{r}\right)$ if $V<\tilde{x}_{r}$, and $m_{r}=V$ if $V \geq \tilde{x}_{r}$. This observation implies that $m_{n}>V+\rho^{1 / 2}$, because if $m_{n} \leq V+\rho^{1 / 2}$ then $m_{r}<V-\rho^{1 / 2}<V$, which is a contradiction. Second, note that $m_{n}>V$ implies $m_{n}<\tilde{x}_{n}$, because $m_{n} \in\left(V, \tilde{x}_{n}\right)$ if $V<\tilde{x}_{n}$, and $m_{n}=V$ if $V \geq \tilde{x}_{n}$. This observation implies that $m_{n}<\tilde{x}_{n}$.

Note that $p\left(\theta_{n}\left(m_{n}\right)\right)\left(m_{n}-V\right) \geq p\left(\theta_{n}\left(m_{n}-\rho^{1 / 2}\right)\right)\left(m_{n}-\rho^{1 / 2}-V\right)$, because $m_{n}$ is the optimal search decision when $J=J_{n}$. Therefore, we have

$$
\begin{aligned}
p\left(\theta_{n}\left(m_{n}\right)\right) \rho^{1 / 2} & \geq\left[p\left(\theta_{n}\left(m_{n}-\rho^{1 / 2}\right)\right)-p\left(\theta_{n}\left(m_{n}\right)\right)\right]\left(m_{n}-\rho^{1 / 2}-V\right) \\
& \geq\left[p\left(\theta_{n}\left(m_{r}\right)\right)-p\left(\theta_{n}\left(m_{r}+\rho^{1 / 2}\right)\right)\right]\left(m_{n}-\rho^{1 / 2}-V\right) \\
& \geq\left[p\left(\theta_{n}\left(m_{r}\right)\right)-p\left(\theta_{n}\left(m_{r}+\rho^{1 / 2}\right)\right)\right]\left(m_{r}+\rho^{1 / 2}-V\right) .
\end{aligned}
$$

To obtain the second inequality we have used the facts that $p\left(\theta_{n}(x)\right)$ is concave in $x$ for all $x \in\left[\underline{x}, \tilde{x}_{n}\right]$, that $m_{r}+\rho^{1 / 2}<m_{n}<\tilde{x}_{n}$, and that $m_{n}-\rho^{1 / 2}-V>0$. To obtain the third inequality we have used the facts that $m_{r}+\rho^{1 / 2}<m_{n}-\rho^{1 / 2}$, and that $p\left(\theta_{n}\left(m_{r}\right)\right)-p\left(\theta_{n}\left(m_{r}+\rho^{1 / 2}\right)\right)>0$. Next, note that $p\left(\theta_{r}\left(m_{r}\right)\right)\left(m_{r}-V\right)$ is greater than $p\left(\theta_{r}\left(m_{r}+\rho^{1 / 2}\right)\right)\left(m_{r}+\rho^{1 / 2}-V\right)$. Therefore, we have

$$
p\left(\theta_{r}\left(m_{r}\right)\right) \rho^{1 / 2} \leq\left[p\left(\theta_{r}\left(m_{r}\right)\right)-p\left(\theta_{r}\left(m_{r}+\rho^{1 / 2}\right)\right)\right]\left(m_{r}+\rho^{1 / 2}-V\right) .
$$

Subtracting this inequality from the previous result and dividing by $\rho^{1 / 2}$, we obtain

$$
\begin{aligned}
& (0<) p\left(\theta_{r}\left(m_{r}\right)\right)-p\left(\theta_{n}\left(m_{n}\right)\right) \\
& \leq \rho^{-1 / 2}\left[p\left(\theta_{r}\left(m_{r}\right)\right)-p\left(\theta_{n}\left(m_{r}\right)\right)+p\left(\theta_{n}\left(m_{r}+\rho^{1 / 2}\right)\right)-p\left(\theta_{r}\left(m_{r}+\rho^{1 / 2}\right)\right)\right]\left(m_{r}+\rho^{1 / 2}-V\right) \\
& <2 p^{\prime}(0) \alpha_{\theta} \rho^{1 / 2}(\bar{x}-\underline{x})=2 \alpha_{R} \rho,
\end{aligned}
$$

where the last line makes use of the fact that the distance between $p\left(\theta_{r}(m)\right)$ and $p\left(\theta_{n}(m)\right)$ is smaller than $p^{\prime}(0) \alpha_{\theta} \rho$, and that $m_{r}+\rho^{1 / 2}-V$ is smaller than $\bar{x}-\underline{x}$.

Overall, we have established that the distance between $p\left(\theta_{r}\left(m_{r}\right)\right)$ and $p\left(\theta_{n}\left(m_{n}\right)\right)$ is such that

$$
\left|p\left(\theta_{r}\left(m_{r}\right)\right)-p\left(\theta_{n}\left(m_{n}\right)\right)\right|<\max \left\{2 \bar{B}_{p} \rho^{1 / 2}+p^{\prime}(0) \alpha_{\theta} \rho, 2 \alpha_{R} \rho^{1 / 2}\right\}=\alpha_{p}(\rho)
$$

Since this result holds for all $(V, y) \in X \times Y$, we conclude that $\left\|\tilde{p}_{r}-\tilde{p}_{n}\right\|<\alpha_{p}(\rho)$. 


\subsection{Unemployment Value}

Given the firm's value function $J \in \mathcal{J}$, the solution to the equilibrium condition (2.7) is the market tightness, $\theta$, defined in (4.1). Given $\theta$, the solution to the equilibrium condition (2.1) is the search value function, $R$, defined as $R(V, y)=\max _{x \in X} f(x, V, y)$. Given $R$, an unemployment value function is a solution to the equilibrium condition (2.2) if and only if it is a fixed point of the mapping $T_{U}$ defined as

$$
\left(T_{U} \varphi\right)(\psi)=v(b)+\beta \mathbb{E}_{\hat{\psi}}\left\{\varphi(\hat{\psi})+\lambda_{u} \max \{0, R(\varphi(\hat{\psi}), \hat{y})\}\right\} .
$$

In the next lemma, we prove that there exists a unique fixed point of the mapping $T_{U}$ within the set $\mathcal{C}(Y)$ of bounded continuous functions $\varphi: Y \rightarrow \mathbb{R}$. Therefore, there exists a unique unemployment value function, $U \in \mathcal{C}(Y)$, that satisfies the equilibrium condition (2.2), and that depends on the aggregate state of the economy, $\psi$, only through the aggregate component of productivity, $y$, but not through the distribution of workers across different employment states, $(u, g)$.

Lemma 4.6. (i) There exists a unique function $U \in \mathcal{C}(Y)$ such that $U=T_{U} U$. (ii) For all $y \in Y$, $U(y) \in[\underline{U}, \bar{U}]$, where $\underline{U}=(1-\beta)^{-1} v(b)>\underline{x}$ and $\bar{U}=v(b)+\beta \bar{x}<\bar{x}$.

Proof: In Appendix C.

Now, consider two arbitrary functions $J_{n}, J_{r} \in \mathcal{J}$. Let $\theta_{n}$ denote the market tightness function computed with $J_{n}, R_{n}$ the search value function computed with $\theta_{n}$, and $U_{n}$ the unemployment value function computed with $R_{n}$. Similarly, let $\theta_{r}$ denote the market tightness function computed with $J_{r}, R_{r}$ the search value function computed with $\theta_{r}$, and $U_{r}$ the unemployment value function computed with $R_{r}$. In the following lemma, we prove that, if the distance between $J_{n}$ and $J_{r}$ converges to zero, so does the distance between $U_{n}$ and $U_{r}$.

Lemma 4.7. For any $\rho>0$ and any $J_{n}, J_{r} \in \mathcal{J}$, if $\left\|J_{n}-J_{r}\right\|<\rho$, then

$$
\left\|U_{n}-U_{r}\right\|<\alpha_{U} \rho, \quad \alpha_{U} \equiv \beta \lambda_{u} \alpha_{R} /(1-\beta) .
$$

ProOF: For the sake of brevity, let us suppress the dependence of various functions on $\hat{y}$. Let $\rho>0$ be an arbitrary real number. Let $J_{n}$ and $J_{r}$ be arbitrary functions in $\mathcal{J}$ such that $\left\|J_{n}-J_{r}\right\|<\rho$. Let $y$ be an arbitrary point in $Y$. The distance between $U_{n}(y)$ and $U_{r}(y)$ is such that

$$
\begin{aligned}
& \left|U_{n}(y)-U_{r}(y)\right| \\
\leq & \beta \mathbb{E}_{\hat{y}}\left\{\left|\left[U_{n}+\lambda_{u} R_{n}\left(U_{n}\right)\right]-\left[U_{r}+\lambda_{u} \max R_{n}\left(U_{r}\right)\right]\right|+\lambda_{u}\left|R_{n}\left(U_{r}\right)-R_{r}\left(U_{r}\right)\right|\right\} \\
< & \beta\left\|U_{n}-U_{r}\right\|+\beta \lambda_{u} \alpha_{R} \rho .
\end{aligned}
$$


To obtain the second inequality we have used the fact that the distance between $U_{n}+\lambda_{u} R_{n}\left(U_{n}\right)$ and $U_{n}+\lambda_{u} R_{n}\left(U_{n}\right)$ is smaller than the distance between $U_{n}$ and $U_{r}$. Since the above result holds for all $y \in Y$, it follows that $\left\|U_{n}-U_{r}\right\|<\alpha_{u} \rho$.

\section{Block Recursive Equilibrium with Dynamic Contracts}

\subsection{Updated Value Function of the Firm}

In the previous section, we have chosen an arbitrary firm's value function, $J \in \mathcal{J}$. Given $J$, we have computed the market tightness function, $\theta$, that satisfies the equilibrium condition (2.7). Given $\theta$, we have computed the search value and policy functions, $R$ and $m$, that satisfy the equilibrium condition (2.1). Given $R$, we have computed the unemployment value function, $U$, that satisfies the equilibrium condition (2.2). In this section, we insert $J, \theta, R, m$ and $U$ into the

right hand side of the equilibrium condition (2.3) to compute an update, $\tilde{J}$, of the firm's value function $J$. This process implicitly defines a mapping $T$ through $\tilde{J}=T J$. More specifically, $\tilde{J}$ is given by ${ }^{18}$

$$
\begin{array}{ll} 
& \tilde{J}(V, y, z) \\
= & \max _{\pi_{i}, w_{i}, \hat{d}_{i}, \hat{V}_{i}} \sum_{i=1}^{2} \pi_{i}\left\{y+z-w_{i}+\beta \mathbb{E}_{\hat{s}}\left[\left(1-d_{i}(\hat{y}, \hat{z})\right)\left(1-\lambda_{e} \tilde{p}\left(\hat{V}_{i}(\hat{y}, \hat{z}), \hat{y}\right)\right) J\left(\hat{V}_{i}(\hat{y}, \hat{z}), \hat{y}, \hat{z}\right)\right]\right\} \\
\text { s.t. } \quad & \pi_{i} \in[0,1], w_{i} \in \mathbb{R}, d_{i}: Y \times Z \rightarrow[\delta, 1], \hat{V}_{i}: Y \times Z \rightarrow X, \quad \text { for } i=1,2, \\
& \sum_{i=1}^{2} \pi_{i}=1, \quad d_{i}(\hat{y}, \hat{z})=\left\{\delta \text { if } U(\hat{y}) \leq \hat{V}_{i}(\hat{y}, \hat{z})+\lambda_{e} R\left(\hat{V}_{i}(\hat{y}, \hat{z}), \hat{y}\right), \quad 1 \text { else }\right\}, \\
& \sum_{i=1}^{2} \pi_{i}\left\{v\left(w_{i}\right)+\beta \mathbb{E}_{\hat{s}}\left[d_{i}(\hat{y}, \hat{z}) U(\hat{y})+\left(1-d_{i}(\hat{y}, \hat{z})\right)\left(\hat{V}_{i}(\hat{y}, \hat{z})+\lambda_{e} R\left(\hat{V}_{i}(\hat{y}, \hat{z}), \hat{y}\right)\right)\right]\right\}=V .
\end{array}
$$

The updated value function of the firm, $\tilde{J}$, has four important properties. First, $\tilde{J}$ depends on the aggregate state of the economy, $\psi$, only through the aggregate component of productivity, $y$, and not through the distribution of workers across different employment states, $(u, g)$. This property follows immediately from the fact that both the objective function and the choice set on the right hand side of (5.1) depend on $y$ but not on $(u, g)$. Second, the updated value function, $\tilde{J}$, is bi-Lipschitz continuous in $V$. More specifically, for all $(y, z) \in Y \times Z$ and all $V_{1}, V_{2} \in X$, with $V_{1} \leq V_{2}$, the difference $\tilde{J}\left(V_{2}, y, z\right)-\tilde{J}\left(V_{1}, y, z\right)$ is bounded between $-\left(V_{2}-V_{1}\right) / \underline{v}^{\prime}$ and $-\left(V_{2}-V_{1}\right) / \bar{v}^{\prime}$ (see part (i) in the proof of Lemma 5.1). Third, $\tilde{J}$ is bounded in $[\underline{J}, \bar{J}]$, where the bounds $\underline{J}$ and

\footnotetext{
${ }^{18}$ In a Block Recursive Equilibrium, the distribution of workers across employment states in the next period, $(\hat{u}, \hat{g})$, is uniquely determined by the realization of the aggregate component of productivity in the next period, $\hat{y}$, and by the state of the economy in the current period, $\psi$. Therefore, in the contracting problem (5.1), next period's separation probability, $d_{i}$, and continuation value, $\hat{V}_{i}$, can be written as functions of $\hat{y}$ only.
} 
$\bar{J}$ are independent of $J$ and $\tilde{J}$ (see (5.2) below). Finally, $\tilde{J}$ is concave in $V$, as a result of the use of the lottery in the contract (see part (iii) in the proof of Lemma 5.1).

The bounds $\underline{B}_{J}, \bar{B}_{J}, \underline{J}$, and $\bar{J}$ are set as

$$
\bar{B}_{J}=\frac{1}{\underline{v}^{\prime}}, \underline{B}_{J}=\frac{1}{\bar{v}^{\prime}},-\underline{J}=\bar{J}=\max \left\{\frac{\left|\bar{y}+\bar{z}-v^{-1}(\underline{x}-\beta \bar{x})\right|}{1-\beta(1-\delta)}, \frac{\left|\underline{y}+\underline{z}-v^{-1}(\bar{x}-\beta \underline{x})\right|}{1-\beta(1-\delta)}\right\} .
$$

With these bounds, $\tilde{J}$ satisfies conditions (J1)-(J3) and, hence, belongs to the set $\mathcal{J}$, as stated in the next lemma.

Lemma 5.1. Set the bounds $\underline{B}_{J}, \bar{B}_{J}, \underline{J}$, and $\bar{J}$ as in (5.2). Then, the updated value function, $\tilde{J}$, belongs to the set $\mathcal{J}$.

Proof: For all $(V, y, z) \in X \times Y \times Z, \tilde{J}(V, y, z)$ is equal to $\max _{\gamma \in \Gamma} F(\gamma, V, y, z)$, where $\gamma$ is defined as the tuple $\left(\pi_{1}, \tilde{V}_{1}, \hat{V}_{1}, \hat{V}_{2}\right) ; \Gamma$ is defined as the set of $\gamma^{\prime}$ s such that $\pi_{1} \in[0,1), \tilde{V}_{1} \in \mathbb{R}$, $\hat{V}_{1}: Y \times Z \rightarrow X$, and $\hat{V}_{2}: Y \times Z \rightarrow X$; and $F(\gamma, V, y, z)$ is defined as

$$
\begin{array}{cl}
F(\gamma, V, y, z) & =\sum_{i=1}^{2} \pi_{i}\left\{y+z-w_{i}+\beta \mathbb{E}_{\hat{s}}\left[\left(1-d_{i}(\hat{y}, \hat{z})\right)\left(1-\lambda_{e} \tilde{p}\left(\hat{V}_{i}(\hat{y}, \hat{z}), \hat{y}\right)\right) J\left(\hat{V}_{i}(\hat{y}, \hat{z}), \hat{y}, \hat{z}\right)\right]\right\}, \\
\text { s.t. } & \pi_{2}=1-\pi_{1}, \quad \tilde{V}_{2}=\left(V-\pi_{1} \tilde{V}_{1}\right) / \pi_{2}, \\
& d_{i}(\hat{y}, \hat{z})=\left\{\delta \text { if } U(\hat{y}) \leq \hat{V}_{i}(\hat{y}, \hat{z})+\lambda_{e} R\left(\hat{V}_{i}(\hat{y}, \hat{z}), \hat{y}\right), 1 \text { else }\right\}, \\
& w_{i}=v^{-1}\left(\tilde{V}_{i}-\beta \mathbb{E}_{\hat{s}}\left[d_{i}(\hat{y}, \hat{z}) U(\hat{y})+\left(1-d_{i}(\hat{y}, \hat{z})\right)\left(\hat{V}_{i}(\hat{y}, \hat{z})+\lambda_{e} R\left(\hat{V}_{i}(\hat{y}, \hat{z}), \hat{y}\right)\right)\right]\right) .
\end{array}
$$

Let $F^{\prime}(\gamma, V, y, z)$ denote the derivative of $F(\gamma, V, y, z)$ with respect to $V$. It is immediate to verify that

$$
F^{\prime}(\gamma, V, y, z)=-\frac{1}{v^{\prime}\left(w_{2}\right)} \in\left[-\frac{1}{\underline{v}^{\prime}},-\frac{1}{\bar{v}^{\prime}}\right]
$$

(i) First, we want to prove that $\tilde{J}$ satisfies property $(\mathrm{J} 1)$ of the set $\mathcal{J}$. To this aim, let $(y, z)$ be an arbitrary point in $Y \times Z$, and let $V_{1}, V_{2}$ be two points in $X$ with $V_{1} \leq V_{2}$. The distance between $\tilde{J}\left(V_{2}, y, z\right)$ and $\tilde{J}\left(V_{1}, y, z\right)$ is such that

$$
\begin{aligned}
\left|\tilde{J}\left(V_{2}, y, z\right)-\tilde{J}\left(V_{1}, y, z\right)\right| & \leq \max _{\gamma \in \Gamma}\left|F\left(\gamma, V_{2}, y, z\right)-F\left(\gamma, V_{1}, y, z\right)\right| \\
& \leq \max _{\gamma \in \Gamma}\left|\int_{V_{1}}^{V_{2}} F^{\prime}(\gamma, t, y, z) d t\right| \\
& \leq \max _{\gamma \in \Gamma} \int_{V_{1}}^{V_{2}}\left|F^{\prime}(\gamma, t, y, z)\right| d t \leq\left|V_{2}-V_{1}\right| \underline{v}^{\prime}
\end{aligned}
$$

The inequality above implies that the function $\tilde{J}$ is Lipschitz continuous in $V$. Therefore, it is absolutely continuous and almost everywhere differentiable with respect to $V$ (see Royden, 
1988, page 112). The function $F$ is differentiable with respect to $V$. Therefore, at any point of differentiability, the derivative of $\tilde{J}$ with respect to $V$ is equal to $F^{\prime}\left(\gamma^{*}(V, y, z), V, y, z\right)$, where $\gamma^{*}(V, y, z)$ belongs to $\arg \max _{\gamma \in \Gamma} F(\gamma, V, y, z)$ (see Milgrom and Segal, 2003, Theorem 1). From these properties of $\tilde{J}$, it follows that the difference $\tilde{J}\left(V_{2}, y, z\right)-\tilde{J}\left(V_{1}, y, z\right)$ is such that

$$
\tilde{J}\left(V_{2}, y, z\right)-\tilde{J}\left(V_{1}, y, z\right)=\int_{V_{1}}^{V_{2}} F^{\prime}\left(\gamma^{*}(t, y, z), t, y, z\right) d t \in\left[-\frac{V_{2}-V_{1}}{\underline{v}^{\prime}},-\frac{V_{2}-V_{1}}{\bar{v}^{\prime}}\right] .
$$

(ii) Next, we want to prove that $\tilde{J}$ satisfies property (J2) of the set $\mathcal{J}$. To this aim, let $(V, y, z)$ be an arbitrary point in $X \times Y \times Z$. Also, let $\gamma_{0}$ denote the tuple $\left(\pi_{1,0}, \tilde{V}_{1,0}, \hat{V}_{1,0}, \hat{V}_{2,0}\right)$, where $\pi_{1,0}=0, \tilde{V}_{1,0}=\bar{x}, \hat{V}_{1,0}=\hat{V}_{2,0}=\underline{x}$. The firm's value $\tilde{J}(V, y, z)$ is such that

$$
\begin{aligned}
\tilde{J}(V, y, z) & \leq \bar{y}+\bar{z}+\beta \delta \bar{J}-\min _{\left(\pi_{i}, \tilde{V}_{i}\right)}\left\{\sum_{i=1}^{2} \pi_{i} v^{-1}\left(\tilde{V}_{i}-\beta \bar{x}\right), \text { s.t. } \sum_{i=1}^{2} \pi_{i} \tilde{V}_{i}=V\right\} \\
& \leq \bar{y}+\bar{z}+\beta \delta \bar{J}-v^{-1}(\underline{x}-\beta \bar{x}) \leq \bar{J}
\end{aligned}
$$

where the first inequality makes use of the bounds on $y, z, w$ and $J$, and the second inequality makes use of the convexity of $v^{-1}($.$) . Also, the firm's value \tilde{J}(V, y, z)$ is such that

$$
\tilde{J}(V, y, z) \geq F\left(\gamma_{0}, V, y, z\right) \geq \underline{y}+\underline{z}-v^{-1}(\bar{x}-\beta \underline{x})+\beta \delta \underline{J} \geq \underline{J},
$$

where the first inequality makes use of the fact that $\gamma_{0}$ belongs to $\Gamma$, and the second inequality makes use of the bounds on $y, z, w$ and $J$.

(iii) In Appendix F, we prove that $\tilde{J}$ is concave with respect to $V$. Hence, $\tilde{J}$ satisfies property (J3) of the set $\mathcal{J}$.

Now, consider two arbitrary functions $J_{n}, J_{r} \in \mathcal{J}$. Let $\theta_{n}, R_{n}, \tilde{p}_{n}, U_{n}, F_{n}$ and $\tilde{J}_{n}$ denote the functions computed with $J_{n}$. Similarly, let $\theta_{r}, R_{r}, \tilde{p}_{r}, U_{r}, F_{r}$ and $\tilde{J}_{r}$ denote the functions computed with $J_{r} \in \mathcal{J}$. The next lemma proves that as the distance between $J_{n}$ and $J_{r}$ converges to zero, the distance between $\tilde{J}_{n}$ and $\tilde{J}_{r}$ converges to zero as well. That is, the mapping $T$ is continuous.

Lemma 5.2. For any $\rho>0$ and any $J_{n}, J_{r} \in \mathcal{J}$, if $\left\|J_{n}-J_{r}\right\|<\rho$, then

$$
\left\|\tilde{J}_{n}-\tilde{J}_{r}\right\|<\beta \lambda_{e} \alpha_{p}(\rho) \bar{J}+\alpha_{J} \rho,
$$

where

$$
\begin{aligned}
& \alpha_{J} \equiv \alpha_{w}+\beta\left[\left(1+\lambda_{e}\right)\left(1+\bar{B}_{J} \alpha_{\hat{V}}\right)+\lambda_{e} \bar{B}_{p} \alpha_{\hat{V}} \bar{J}\right] \\
& \alpha_{\hat{V}} \equiv\left(\lambda_{e} \alpha_{R}+\alpha_{U}+1\right) /\left(1-\lambda_{e}\right), \quad \alpha_{w} \equiv \beta\left(\alpha_{U}+\alpha_{\hat{V}}+\lambda_{e} \alpha_{R}\right) / \underline{v}^{\prime} .
\end{aligned}
$$


ProOF: For the sake of brevity, suppress the dependence of various functions on $(\hat{y}, \hat{z})$. Let $\rho>0$ be an arbitrary real number. Let $J_{n}$ and $J_{r}$ be arbitrary functions in $\mathcal{J}$ such that $\left\|J_{n}-J_{r}\right\|<$ $\rho$. Let $(V, y, z)$ to an arbitrary point in $X \times Y \times Z$. Without loss in generality, assume that $\tilde{J}_{n}(V, y, z) \leq \tilde{J}_{r}(V, y, z)$. (If $\tilde{J}_{n}(V, y, z)>\tilde{J}_{r}(V, y, z)$, just switch the roles of $\tilde{J}_{n}$ and $\tilde{J}_{r}$ in the proof below).

Denote as $\gamma_{r}=\left(\pi_{1, r}, \tilde{V}_{1, r}, \hat{V}_{1, r}, \hat{V}_{2, r}\right)$ a tuple such that $\gamma_{r} \in \Gamma$ and $F_{r}\left(\gamma_{r}, V, y, z\right)=\tilde{J}_{r}(V, y, z)$. Also, denote as $w_{i, r}$ and $d_{i, r}$ the wage and the separation probability implied by (5.3) for $F(\gamma, V, y, z)=$ $F_{r}\left(\gamma_{r}, V, y, z\right)$. Denote as $\gamma_{n}$ the tuple $\left(\pi_{1, n}, \tilde{V}_{1, n}, \hat{V}_{1, n}, \hat{V}_{2, n}\right)$, where $\pi_{1, n}=\pi_{1, r}, \tilde{V}_{1, n}=\tilde{V}_{1, r}$, and

$$
\hat{V}_{i, n}=\left\{\begin{array}{l}
\hat{V}_{i, r}, \text { if }\left[\hat{V}_{i, r}+\lambda_{e} R_{n}\left(\hat{V}_{i, r}\right)-U_{n}\right]\left[\hat{V}_{i, r}+\lambda_{e} R_{r}\left(\hat{V}_{i, r}\right)-U_{r}\right]>0, \\
U_{n}-\lambda_{e} R_{n}\left(\hat{V}_{i, n}\right)+\rho, \text { if } \hat{V}_{i, r}+\lambda_{e} R_{n}\left(\hat{V}_{i, r}\right) \leq U_{n}, \hat{V}_{i, r}+\lambda_{e} R_{r}\left(\hat{V}_{i, r}\right) \geq U_{r}, \\
U_{n}-\lambda_{e} R_{n}\left(\hat{V}_{i, n}\right)-\rho, \text { if } \hat{V}_{i, r}+\lambda_{e} R_{n}\left(\hat{V}_{i, r}\right) \geq U_{n}, \hat{V}_{i, r}+\lambda_{e} R_{r}\left(\hat{V}_{i, r}\right)<U_{r} .
\end{array}\right.
$$

Let $w_{i, n}$ and $d_{i, n}$ denote the wage and separation probability implied by $(5.3)$ for $F(\gamma, V, y, z)=$ $F_{n}\left(\gamma_{n}, V, y, z\right)$. Note that (5.5) implies $d_{i, n}=d_{i, r}$.

First, we want to bound the distance $\left\|\hat{V}_{i, n}-\hat{V}_{i, r}\right\|$. To this aim, let $(\hat{y}, \hat{z})$ denote an arbitrary point in $Y \times Z$. Consider the case in which $\hat{V}_{i, r}+\lambda_{e} R_{n}\left(\hat{V}_{i, r}\right)-U_{n}$ has the same sign as $\hat{V}_{i, r}+$ $\lambda_{e} R_{r}\left(\hat{V}_{i, r}\right)-U_{r}$. In this case, $\hat{V}_{i, n}=\hat{V}_{i, r}$ and, hence, $\left\|\hat{V}_{i, n}-\hat{V}_{i, r}\right\|<\alpha_{\hat{V}} \rho$. Next, consider the case in which $\hat{V}_{i, r}+\lambda_{e} R_{n}\left(\hat{V}_{i, r}\right)-U_{n}$ has a different sign from $\hat{V}_{i, r}+\lambda_{e} R_{r}\left(\hat{V}_{i, r}\right)-U_{r}$. In this case, the absolute value of $\hat{V}_{i, r}+\lambda_{e} R_{n}\left(\hat{V}_{i, r}\right)-U_{n}$ is such that

$$
\begin{aligned}
\left|\hat{V}_{i, r}+\lambda_{e} R_{n}\left(\hat{V}_{i, r}\right)-U_{n}\right| & \leq\left|\hat{V}_{i, r}+\lambda_{e} R_{n}\left(\hat{V}_{i, r}\right)-U_{n}-\left(\hat{V}_{i, r}+\lambda_{e} R_{r}\left(\hat{V}_{i, r}\right)-U_{r}\right)\right| \\
& \leq\left(\lambda_{e} \alpha_{R}+\alpha_{U}\right) \rho,
\end{aligned}
$$

where the second inequality makes use of the bounds in (4.9) and (4.12). Moreover, the absolute value of $\hat{V}_{i, r}+\lambda_{e} R_{n}\left(\hat{V}_{i, r}\right)-U_{n}$ is such that

$$
\begin{aligned}
\left|\hat{V}_{i, r}+\lambda_{e} R_{n}\left(\hat{V}_{i, r}\right)-U_{n}\right| & =\left|\hat{V}_{i, r}+\lambda_{e} R_{n}\left(\hat{V}_{i, r}\right)-U_{n}-\left(\hat{V}_{i, n}+\lambda_{e} R_{n}\left(\hat{V}_{i, n}\right)-U_{n}\right)\right|-\rho \\
& \geq\left(1-\lambda_{e}\right)\left|\hat{V}_{i, r}-\hat{V}_{i, n}\right|-\rho,
\end{aligned}
$$

where the equality makes use of the definition of $\hat{V}_{i, n}$ in (5.5), and the inequality makes use of the bounds in (4.6). From (5.6) and (5.7), it follows that $(0<) \hat{V}_{i, n}-\hat{V}_{i, r}<\alpha_{\hat{V}} \rho$ and, hence, $\left|\hat{V}_{i, n}-\hat{V}_{i, r}\right|<\alpha_{\hat{V}} \rho$. Since these results hold for all $(\hat{y}, \hat{z}) \in Y \times Z$, we have

$$
\left\|\hat{V}_{i, n}-\hat{V}_{i, r}\right\|<\alpha_{\hat{V}} \rho
$$

Second, we want to bound the distance $\left|w_{i, r}-w_{i, n}\right|$. From the definitions of $w_{i, r}$ and $w_{i, n}$, it 
follows that $v\left(w_{i, r}\right)$ and $v\left(w_{i, n}\right)$ are

$$
\begin{aligned}
v\left(w_{i, r}\right) & =\tilde{V}_{i, r}-\beta \mathbb{E}_{\hat{s}}\left[d_{i, r} U_{r}+\left(1-d_{i, r}\right)\left(\hat{V}_{i, r}+\lambda_{e} R_{r}\left(\hat{V}_{i, r}\right)\right)\right] \\
v\left(w_{i, n}\right) & =\tilde{V}_{i, n}-\beta \mathbb{E}_{\hat{s}}\left[d_{i, n} U_{n}+\left(1-d_{i, n}\right)\left(\hat{V}_{i, n}+\lambda_{e} R_{n}\left(\hat{V}_{i, n}\right)\right)\right] \\
& =\tilde{V}_{i, r}-\beta \mathbb{E}_{\hat{s}}\left[d_{i, r} U_{n}+\left(1-d_{i, r}\right)\left(\hat{V}_{i, n}+\lambda_{e} R_{n}\left(\hat{V}_{i, n}\right)\right)\right],
\end{aligned}
$$

where the last line makes use of the fact that, by construction, $\tilde{V}_{i, n}=\tilde{V}_{i, r}$ and $d_{i, n}=d_{i, r}$. From the previous equations, it follows that the distance between $v\left(w_{i, n}\right)$ and $v\left(w_{i, r}\right)$ is such that

$$
\begin{aligned}
\left|v\left(w_{i, n}\right)-v\left(w_{i, r}\right)\right| & \geq \underline{v}^{\prime}\left|w_{i, n}-w_{i, r}\right| \\
\left|v\left(w_{i, n}\right)-v\left(w_{i, r}\right)\right| & \leq \beta \mathbb{E}_{\hat{s}}\left\{\left|U_{n}-U_{r}\right|+\left|\left[\hat{V}_{i, n}+\lambda_{e} R_{n}\left(\hat{V}_{i, n}\right)\right]-\left[\hat{V}_{i, r}+\lambda_{e} R_{n}\left(\hat{V}_{i, r}\right)\right]\right|\right\} \\
& +\beta \mathbb{E}_{\hat{s}}\left\{\left|\left[\hat{V}_{i, r}+\lambda_{e} R_{n}\left(\hat{V}_{i, r}\right)\right]-\left[\hat{V}_{i, r}+\lambda_{e} R_{r}\left(\hat{V}_{i, r}\right)\right]\right|\right\} \\
& <\beta\left(\alpha_{U}+\alpha_{\hat{V}}+\lambda_{e} \alpha_{R}\right) \rho,
\end{aligned}
$$

where the last inequality makes use of the bounds in (4.12), (5.8) and (4.9). Taken together, the two inequalities in (5.9) imply that

$$
\left|w_{i, n}-w_{i, r}\right|<\alpha_{w} \rho
$$

Third, we want to bound the distance between $\left(1-\lambda_{e} \tilde{p}_{n}\left(\hat{V}_{i, n}\right)\right) J_{n}\left(\hat{V}_{i, n}\right)$ and $\left(1-\lambda_{e} \tilde{p}_{r}\left(\hat{V}_{i, r}\right)\right) J_{r}\left(\hat{V}_{i, r}\right)$ To this aim, note that the distance between $J_{n}\left(\hat{V}_{i, n}\right)$ and $J_{r}\left(\hat{V}_{i, r}\right)$ is such that

$$
\left|J_{n}\left(\hat{V}_{i, n}\right)-J_{r}\left(\hat{V}_{i, r}\right)\right| \leq\left|J_{n}\left(\hat{V}_{i, n}\right)-J_{n}\left(\hat{V}_{i, r}\right)\right|+\left|J_{n}\left(\hat{V}_{i, r}\right)-J_{r}\left(\hat{V}_{i, r}\right)\right|<\left(1+\bar{B}_{J} \alpha_{\hat{V}}\right) \rho,
$$

where the last inequality makes use of the bounds in (5.8). Also, note that the distance between $\tilde{p}_{n}\left(\hat{V}_{i, n}\right) J_{n}\left(\hat{V}_{i, n}\right)$ and $\tilde{p}_{r}\left(\hat{V}_{i, r}\right) J_{r}\left(\hat{V}_{i, r}\right)$ is such that

$$
\begin{aligned}
& \left|\tilde{p}_{n}\left(\hat{V}_{i, n}\right) J_{n}\left(\hat{V}_{i, n}\right)-\tilde{p}_{r}\left(\hat{V}_{i, r}\right) J_{r}\left(\hat{V}_{i, r}\right)\right| \\
\leq \quad & \tilde{p}_{n}\left(\hat{V}_{i, n}\right)\left|J_{n}\left(\hat{V}_{i, n}\right)-J_{r}\left(\hat{V}_{i, n}\right)\right|+\tilde{p}_{n}\left(\hat{V}_{i, n}\right)\left|J_{r}\left(\hat{V}_{i, n}\right)-J_{r}\left(\hat{V}_{i, r}\right)\right| \\
& +\left|J_{r}\left(\hat{V}_{i, r}\right)\right|\left|\tilde{p}_{n}\left(\hat{V}_{i, n}\right)-\tilde{p}_{n}\left(\hat{V}_{i, r}\right)\right|+\left|J_{r}\left(\hat{V}_{i, r}\right)\right|\left|\tilde{p}_{n}\left(\hat{V}_{i, r}\right)-\tilde{p}_{r}\left(\hat{V}_{i, r}\right)\right| \\
< & \left(1+\bar{B}_{J} \alpha_{\hat{V}}+\bar{B}_{p} \alpha_{\hat{V}} \bar{J}\right) \rho+\alpha_{p}(\rho) \bar{J},
\end{aligned}
$$

where we have used Lemma 4.10 to bound the last difference. From (5.11) and (5.12), it follows that

$$
\begin{aligned}
& \left|\left(1-\lambda_{e} \tilde{p}_{n}\left(\hat{V}_{i, n}\right)\right) J_{n}\left(\hat{V}_{i, n}\right)-\left(1-\lambda_{e} \tilde{p}_{r}\left(\hat{V}_{i, r}\right)\right) J_{r}\left(\hat{V}_{i, r}\right)\right| \\
\leq & \left|J_{n}\left(\hat{V}_{i, n}\right)-J_{r}\left(\hat{V}_{i, r}\right)\right|+\lambda_{e}\left|\tilde{p}_{n}\left(\hat{V}_{i, n}\right) J_{n}\left(\hat{V}_{i, n}\right)-\tilde{p}_{r}\left(\hat{V}_{i, r}\right) J_{r}\left(\hat{V}_{i, r}\right)\right| \\
< & \lambda_{e} \alpha_{p}(\rho) \bar{J}+\left[\left(1+\lambda_{e}\right)\left(1+\bar{B}_{J} \alpha_{\hat{V}}\right)+\lambda_{e} \bar{B}_{p} \alpha_{\hat{V}} \bar{J}\right] \rho .
\end{aligned}
$$


Finally, we want to bound the distance between $\tilde{J}_{r}(V, y, z)$ and $\tilde{J}_{n}(V, y, z)$. To this aim, note that the difference $\tilde{J}_{r}(V, y, z)-\tilde{J}_{n}(V, y, z)$ is positive (by assumption). Also, note that the difference $\tilde{J}_{r}(V, y, z)-\tilde{J}_{n}(V, y, z)$ is smaller than $F_{r}\left(\gamma_{r}, V, y, z\right)-F_{n}\left(\gamma_{n}, V, y, z\right)$ because $\tilde{J}_{r}(V, y, z)=$ $F_{r}\left(\gamma_{r}, V, y, z\right)$ and $F_{n}\left(\gamma_{n}, V, y, z\right) \leq \tilde{J}_{n}(V, y, z)$. From these observations and the bounds (5.8), (5.10) and (5.13), it follows that

$$
\begin{aligned}
0 & \leq \tilde{J}_{r}(V, y, z)-\tilde{J}_{n}(V, y, z) \leq F_{r}\left(\gamma_{r}, V, y, z\right)-F_{n}\left(\gamma_{n}, V, y, z\right) \\
& \leq \sum_{i=1}^{2} \pi_{i, r}\left\{\left|w_{i, n}-w_{i, r}\right|+\beta \mathbb{E}_{\hat{s}}\left[\left|\left(1-\lambda_{e} \tilde{p}_{n}\left(\hat{V}_{i, n}\right)\right) J_{n}\left(\hat{V}_{i, n}\right)-\left(1-\lambda_{e} \tilde{p}_{r}\left(\hat{V}_{i, r}\right)\right) J_{r}\left(\hat{V}_{i, r}\right)\right|\right]\right\} \\
& <\beta \lambda_{e} \alpha_{p}(\rho) \bar{J}+\left\{\alpha_{w}+\beta\left[\left(1+\lambda_{e}\right)\left(1+\bar{B}_{J} \alpha_{\hat{V}}\right)+\lambda_{e} \bar{B}_{p} \alpha_{\hat{V}} \bar{J}\right]\right\} \rho,
\end{aligned}
$$

Since the above inequality holds for all $(V, y, z) \in X \times Y \times Z$, it implies the result stated in the lemma.

\subsection{Existence of a Block Recursive Equilibrium}

Now, we are in the position to establish the paper's main result.

\section{Theorem 5.3. There exists a Block Recursive Equilibrium.}

Proof: First, fix $\varepsilon>0$ to be an arbitrary real number. Let $\rho_{\varepsilon}$ be the unique positive solution for $\rho$ of the equation $\beta \lambda_{e} \alpha_{p}(\rho) \bar{J}+\alpha_{j} \rho=\varepsilon$. For all $J_{n}, J_{r} \in \mathcal{J}$ such that $\left\|J_{n}-J_{r}\right\|<\rho_{\varepsilon}$, Lemma 5.2 implies that $\left\|T J_{n}-T J_{r}\right\|<\varepsilon$. Hence, the equilibrium operator $T$ is continuous. Next, let $\rho_{y}$ denote the minimum distance between distinct elements of the set $Y$, and $\rho_{z}$ the minimum distance between distinct elements of the set $Z$, i.e. $\rho_{y}=\min _{Y}\left|y_{i}-y_{j}\right|$ and $\rho_{z}=\min _{Z}\left|z_{i}-z_{j}\right| \cdot{ }^{19}$ Also, let $\|\cdot\|_{E}$ denote the standard norm on the Euclidean space $X \times Y \times Z$. Let $\tilde{\rho}_{\varepsilon}=\min \left\{\underline{v}^{\prime} \varepsilon, \rho_{y}, \rho_{z}\right\}$. For all $\left(V_{1}, y_{1}, z_{1}\right),\left(V_{2}, y_{2}, z_{2}\right) \in X \times Y \times Z$ such that $\left\|\left(V_{2}, y_{2}, z_{2}\right)-\left(V_{1}, y_{1}, z_{1}\right)\right\|_{E}<\tilde{\rho}_{\varepsilon}$ and all $J \in \mathcal{J}$, Lemma 5.1 implies that $T J$ satisfies the property (J1) of the set $\mathcal{J}$ and, consequently, $\left|(T J)\left(V_{2}, y_{2}, z_{2}\right)-(T J)\left(V_{2}, y_{2}, z_{2}\right)\right|<\varepsilon$. Hence, the family of functions $T(\mathcal{J})$ is equicontinuous. Finally, Lemma 5.1 implies that the equilibrium operator $T$ maps the set of functions $\mathcal{J}$ into itself.

From these properties, it follows that the equilibrium operator $T$ satisfies the conditions of Schauder's fixed point theorem (Stokey and Lucas with Prescott, 1989, Theorem 17.4). Therefore, there exists a firm's value function $J^{*} \in \mathcal{J}$ such that $T J^{*}=J^{*}$. Denote as $\theta^{*}$ the market tightness function computed with $J^{*}$. Denote as $R^{*}$ and $m^{*}$ the search value and policy functions computed with $\theta^{*}$. Denote as $U^{*}$ the unemployment value function computed with $R^{*}$.

\footnotetext{
${ }^{19}$ If $Y$ contains only one element, we can set $\rho_{y}=1$. Similarly, if $Z$ contains only one elemet, set $\rho_{z}=1$.
} 
Denote as $c^{*}$ the contract policy function computed with $J^{*}, \theta^{*}, R^{*}, m^{*}$, and $U^{*}$. The functions $\left\{\theta^{*}, R^{*}, m^{*}, U^{*}, J^{*}, c^{*}\right\}$ satisfy the conditions (i)-(v) in the definition of a recursive equilibrium. The functions $\left\{\theta^{*}, R^{*}, m^{*}, U^{*}, J^{*}, c^{*}\right\}$ depend on the aggregate state of the economy, $\psi$, only through the aggregate component of productivity, $y$, and not through the distribution of workers across different employment states, $(u, g)$. Hence, the functions $\left\{\theta^{*}, R^{*}, m^{*}, U^{*}, J^{*}, c^{*}\right\}$ constitute a Block Recursive Equilibrium.

Directed search is necessary for existence of a Block Recursive Equilibrium. To see this necessity clearly, suppose that search is random. Then the equilibrium condition (2.7) is replaced by

$$
k \geq \max _{x \in X} q(\theta(\psi)) \mathbb{I}(x, \psi) J\left(x, \psi, z_{0}\right),
$$

and $\theta(\psi) \geq 0$, with complementary slackness. The term on the LHS of (5.14) is the cost of creating a vacancy. The expression on the RHS of (5.14) is the maximized benefit of creating a vacancy. The first term on the RHS is the probability that a firm meets a worker. The second term denotes the probability that a worker met by a firm is willing to accept an employment contract that provides him with the lifetime utility $x$. The third term is the value to the firm of being matched with a worker to whom it has promised the lifetime utility $x$. With random search, $x$ is a random draw from the offer distribution, and so a worker's acceptance probability of a new match depends on the distribution of workers across employment states. The equilibrium condition (5.14) holds only if the distribution affects also the equilibrium market tightness or the firm's value function. In either case, the equilibrium fails to be block recursive with random search. In contrast, directed search eliminates the dependence of the acceptance probability on the distribution of workers because a worker always accepts a job that he chooses to search for; that is, $\mathbb{I}\left(x^{*}, \psi\right)=1$ where $x^{*}=m(V, \psi) \cdot{ }^{20}$

For the sake of completeness, let us list three other assumptions about the production technology and the search process that are necessary for existence of a Block Recursive Equilibrium: the linear production function, the vacancy cost independent of the aggregate vacancy rate, and a matching technology with constant returns to scale. If the production function were either concave or convex, the distribution of workers across different employment states would affect the output of a match and, in turn, the firm's value function, the market tightness function and the value of unemployment. If the vacancy cost depends the aggregate vacancy rate, the distribution of workers across different employment states would affect the aggregate vacancy rate, the vacancy

\footnotetext{
${ }^{20}$ When $m(V, \psi)$ is strictly increasing in $V$, the workers are endogenously separated into different submarkets in the search process. This endogenous separation is a common feature of directed search (e.g., Moen, 1997, and Acemoglu and Shimer, 1999), and it also holds in the presence of ex ante heterogeneity (e.g., Shi, 2001).
} 
cost and, ultimately, the equilibrium market tightness. Finally, if the matching process between vacancies and applicants exhibits non-constant returns to scale, the distribution of applicants across different submarkets (and, hence, the distribution of workers across different employment states) would affect the market tightness function and, in turn, the firm's and worker's value functions. We emphasize that these assumptions are standard and that they alone do not lead to block recursivity of an equilibrium. For example, they are maintained in the models of search on the job by Burdett and Mortensen (1998), Postel-Vinay and Robin (2002), and Burdett and Coles (2003), where the equilibrium fails to be block recursive because search is undirected.

\section{Block Recursive Equilibrium with Fixed-Wage Contracts}

In the model with fixed-wage contracts, the equilibrium operator $T$ may not be continuous. For example, the search value function, $R_{n}$, and the unemployment value function, $U_{n}$, computed with the firm's value function $J_{n}$ may be such that the worker prefers being employed at the wage $w$ than being unemployed. However, given a firm's value function $J_{r}$ that is arbitrarily close to $J_{n}$, the search value function, $R_{r}$, and the unemployment value function, $U_{r}$, may be such that the worker's preference ordering between employment at the wage $w$ and unemployment is reversed. When this is the case, the probability with which a worker leaves a job that pays the wage $w$ is not continuous in $J$ and, hence, the firm's value from employing a worker at the wage $w, K(w, s)$ defined in (2.5), and the firm's updated value function, $T J$, are not continuous in $J .^{21}$

Since the equilibrium operator $T$ may not be continuous, we cannot appeal to Schauder's theorem in order to prove existence of a fixed point of $T$, and, in turn, existence of a Block Recursive Equilibrium. Instead, we adopt the following strategy. We consider a proxy of the model with fixed-wage contracts in which a worker is not allowed to voluntarily quit his jobs during the separation stage. Formally, in this proxy model, the equilibrium conditions (2.4) and (2.5) are replaced by

$$
H(w, \psi)=w+\beta \mathbb{E}_{\hat{\psi}}\left\{\delta U(\hat{\psi})+(1-\delta)\left[H(w, \hat{\psi})+\lambda_{e} \max \{0, R(H(w, \hat{\psi}), \hat{\psi})\}\right]\right\}
$$

and

$$
K(w, s)=y+z-w+\beta(1-\delta) \mathbb{E}_{\hat{s}}\left[\left(1-\lambda_{e} \tilde{p}(H(w, \hat{\psi}), \hat{\psi})\right) K(H(w, \hat{\psi}), \hat{s})\right] .
$$

We prove that the equilibrium operator associated with the proxy model admits a fixed point because it satisfies all the conditions of Schauder's theorem (including continuity). Finally, we

\footnotetext{
${ }^{21}$ This discontinuity does not occur with dynamic contracts, because the future wage path (i.e., the promised future value) can be adjusted to ensure that job separation rates are close to each other whenever the firm's value functions are close to each other. See the proof of Lemma 5.2.
} 
use the fixed point to construct a Block Recursive Equilibrium of the proxy model. If, along the equilibrium path, a worker never has the incentive to quit his job during the separation stage, the BRE of the proxy model is also a BRE of the original model.

\subsection{Employment Value}

Given an arbitrary firm's value function $J \in \mathcal{J}$, let $R$ denote the search value function that solves the equilibrium condition (2.1), and $U$ the unemployment value function that solves the equilibrium condition (2.2). Given $R$ and $U$, an employment value function is a solution to the equilibrium condition (6.1) if and only if it is a fixed point of the mapping $T_{H}$ defined as

$$
\left(T_{H} \varphi\right)(w, \psi)=w+\beta \mathbb{E}_{\hat{\psi}}\left\{\delta U(\hat{y})+(1-\delta)\left[\varphi(w, \hat{\psi})+\lambda_{e} \max \{0, R(\varphi(w, \hat{\psi}), \hat{y})\}\right]\right\} .
$$

In Lemma 6.1, we prove that there exists a unique fixed point of the mapping $T_{H}$ within the set $\mathcal{C}(W \times Y)$ of bounded continuous functions $\varphi: W \times Y \rightarrow \mathbb{R}$ (where $W$ is defined below). Therefore, there exists a unique employment value function, $H$, that satisfies the equilibrium condition (6.1), and depends on the aggregate state of the economy, $\psi$, only through the aggregate component of productivity, $y$. Moreover, in Lemma 6.1, we prove that $H$ is strictly decreasing and bi-Lipschitz continuous in $w$.

Lemma 6.1. Let $W=[\underline{w}, \bar{w}]$, where $\bar{w}$ is given by $[1-\beta(1-\delta)] \bar{x}-\beta \delta \underline{U}$ and $\underline{w}$ by $\underline{x}-\beta[1-$ $\beta(1-\delta)]^{-1}(\bar{w}+\beta \delta \bar{U})$. (i) There exists a unique function $H \in \mathcal{C}(W \times Y)$ such that $H=T_{H} H$.

(ii) For all $y \in Y$ and all $w_{1}, w_{2} \in W, w_{1} \leq w_{2}, H$ is such that

$$
w_{2}-w_{1} \leq H\left(w_{2}, y\right)-H\left(w_{1}, y\right) \leq\left(w_{2}-w_{1}\right) /[1-\beta(1-\delta)]
$$

(iii) For all $y \in Y, H$ is such that

$$
H(\underline{w}, y) \leq \underline{x}, \quad \bar{x} \leq H(\bar{w}, y), \quad \text { all } y \in Y .
$$

Proof: In Appendix D.

From the properties of the employment value function, $H$, we can derive some properties of the wage function, $h$, which is the solution of the equation $H(w, \psi)=V$ with respect to $w$. First, since $H$ is strictly increasing in $w, h$ is well-defined. Second, since $H$ is strictly increasing and bi-Lipschitz continuous in $w, h$ is strictly increasing and bi-Lipschitz in $V$. More specifically, for all $y \in Y$ and all $V_{1}, V_{2} \in X$, with $V_{1} \leq V_{2}$, we have

$$
[1-\beta(1-\delta)]\left(V_{2}-V_{1}\right) \leq h\left(V_{2}, y\right)-h\left(V_{1}, y\right) \leq V_{2}-V_{1} \text {. }
$$


Finally, since $H$ is strictly increasing in $w$ and satisfies property $(6.5), h(V, y)$ belongs to the interval $W$ for all $(V, y) \in X \times Y$.

Now, consider two arbitrary functions $J_{n}, J_{r} \in \mathcal{J}$. Let $R_{n}, U_{n}, H_{n}$ and $h_{n}$ denote the functions computed with $J_{n}$. Similarly, let $R_{r}, U_{r}, H_{r}$ and $h_{r}$ denote the functions computed with $J_{r} \in \mathcal{J}$. Lemma 6.2 proves that as the distance between $J_{n}$ and $J_{r}$ converges to zero, the distance between $H_{n}$ and $H_{r}$ and the distance between $h_{n}$ and $h_{r}$ both converge to zero. That is, $H$ and $h$ are continuous in $J$.

Lemma 6.2. For any $\rho>0$ and any $J_{n}, J_{r} \in \mathcal{J}$, if $\left\|J_{n}-J_{r}\right\|<\rho$, then

$$
\begin{aligned}
& \left\|H_{n}-H_{r}\right\|<\alpha_{h} \rho, \quad\left\|h_{n}-h_{r}\right\|<\alpha_{h} \rho, \\
& \alpha_{h} \equiv \beta\left(\alpha_{u}+\lambda_{e} \alpha_{R}\right) /(1-\beta) .
\end{aligned}
$$

Proof: Let $\rho>0$ be an arbitrary real number; let $J_{n}$ and $J_{r}$ be arbitrary functions in $\mathcal{J}$ such that $\left\|J_{n}-J_{r}\right\|<\rho$. Let $(w, y)$ be an arbitrary point in $W \times Y$. Then, the distance between $H_{n}(w, y)$ and $H_{r}(w, y)$ is such that

$$
\begin{aligned}
& \left|H_{n}(w, y)-H_{r}(w, y)\right| \\
\leq & \beta \mathbb{E}_{\hat{y}}\left\{\left|U_{n}(y)-U_{r}(y)\right|+\lambda_{e}\left|\max \left\{0, R_{n}\left(H_{n}(w, \hat{y}), \hat{y}\right)\right\}-\max \left\{0, R_{r}\left(H_{n}(w, \hat{y}), \hat{y}\right)\right\}\right|\right\} \\
+ & \beta \mathbb{E}_{\hat{y}}\left\{\left|H_{n}(w, \hat{y})+\lambda_{e} \max \left\{0, R_{r}\left(H_{n}(w, \hat{y}), \hat{y}\right)\right\}-H_{r}(w, \hat{y})-\lambda_{e} \max \left\{0, R_{r}\left(H_{r}(w, \hat{y}), \hat{y}\right)\right\}\right|\right\} \\
< & \beta\left(\alpha_{u}+\lambda_{e} \alpha_{R}\right) \rho+\beta\left\|H_{n}-H_{r}\right\|,
\end{aligned}
$$

where the last inequality makes use of the bounds in (4.12), (4.9), and (4.6). Since the above result holds for all $(w, y) \in W \times Y$, the RHS is an upper bound on $\left\|H_{n}-H_{r}\right\|$. Re-arranging terms yields the bound on $\left\|H_{n}-H_{r}\right\|$ given by (6.7).

Now, let $(V, y)$ be an arbitrary point in $X \times Y$. The distance between $h_{n}(V, y)$ and $h_{r}(V, y)$ is such that

$$
\begin{aligned}
\left|h_{n}(V, y)-h_{r}(V, y)\right| & \leq\left|H_{n}\left(h_{n}(V, y), y\right)-H_{n}\left(h_{r}(V, y), y\right)\right| \\
& =\left|H_{r}\left(h_{r}(V, y), y\right)-H_{n}\left(h_{r}(V, y), y\right)\right|<\alpha_{h} \rho,
\end{aligned}
$$

where the first inequality uses the fact that $H_{n}(w, y)$ satisfies condition (6.4), and the equality uses the fact that $H_{n}\left(h_{n}(V, y), y\right)=H_{r}\left(h_{r}(V, y), y\right)=V$. Since the above result holds for all $(V, y) \in X \times Y$, the RHS is an upper bound on $\left\|h_{n}-h_{r}\right\|$, as given by (6.7).

\subsection{Value Function of the Firm}

Let $H$ and $\tilde{p}$ denote the employment value function and the separation probability computed with an arbitrary function $J \in \mathcal{J}$. Given $H$ and $\tilde{p}$, a firm's value function is a solution to the 
equilibrium condition (6.2) if and only if it is a fixed point of the mapping $T_{K}$ defined as

$$
\left(T_{K} \varphi\right)(w, s)=y+z-w+\beta(1-\delta) \mathbb{E}_{\hat{s}}\left[\left(1-\lambda_{e} \tilde{p}(H(w, \hat{y}), \hat{y})\right) \varphi(w, \hat{s})\right]
$$

In Lemma 6.3, we prove that there exists a unique fixed point of the mapping $T_{K}$ within the set $\mathcal{C}(W \times Y \times Z)$ of bounded continuous functions $\varphi: W \times Y \times Z \rightarrow \mathbb{R}$. Therefore, there exists a unique firm's value function, $K$, that satisfies the equilibrium condition (6.2), and that depends on the aggregate state of the economy, $\psi$, only through the aggregate component of productivity, $y$. Then, we prove that $K$ is bounded between $\underline{K}$ and $\bar{K}$, where

$$
-\underline{K}=\bar{K}=\max \left\{\frac{|\underline{y}+\underline{z}-\bar{w}|}{1-\beta(1-\delta)}, \frac{|\bar{y}+\bar{z}-\underline{w}|}{1-\beta(1-\delta)}\right\} .
$$

Finally, we prove that $K$ is bi-Lipschitz continuous in $w$. That is, for all $w_{1} \leq w_{2}$, the difference $K\left(w_{2}, y, z\right)-K\left(w_{1}, y, z\right)$ is bounded between $-\bar{B}_{K}\left(w_{2}-w_{1}\right)$ and $-\underline{B}_{K}\left(w_{2}-w_{1}\right)$, where

$$
\underline{B}_{K}=\frac{1-\beta(1-\delta)\left(1+\lambda_{e} \bar{B}_{p} \bar{K}\right)}{[1-\beta(1-\delta)]\left[1-\beta(1-\delta)\left(1-\lambda_{e}\right)\right]}, \bar{B}_{K}=\frac{1-\beta(1-\delta)\left(1+\lambda_{e} \bar{B}_{p} \underline{K}\right)}{[1-\beta(1-\delta)]^{2}},
$$

In the remainder of this section, we will assume that the parameters of the model are such that $0<\underline{B}_{K} \leq \bar{B}_{K}<\infty .^{22}$

Lemma 6.3. (i) There exists a unique function $K \in \mathcal{C}(W \times Y \times Z)$ such that $K=T_{K} K$. (ii) For all $(y, z) \in Y \times Z$ and all $w_{1}, w_{2} \in W$, with $w_{1} \leq w_{2}, K$ is such that

$$
-\bar{B}_{K}\left(w_{2}-w_{1}\right) \leq K\left(w_{2}, y, z\right)-K\left(w_{1}, y, z\right) \leq-\underline{B}_{K}\left(w_{2}-w_{1}\right)
$$

(iii) For all $(w, y, z) \in W \times Y \times Z, K$ is such that

$$
K(w, y, z) \in[\underline{K}, \bar{K}]
$$

Proof: In Appendix E.

Now, consider two arbitrary functions $J_{n}, J_{r} \in \mathcal{J}$. Let $R_{n}, U_{n}, H_{n}, h_{n}$ and $K_{n}$ denote the functions computed with $J_{n}$. Similarly, let $R_{r}, U_{r}, H_{r}, h_{r}$ and $K_{r}$ denote the functions computed with $J_{r} \in \mathcal{J}$. Lemma 6.4 proves that as the distance between $J_{n}$ and $J_{r}$ converges to zero, the distance between $K_{n}$ and $K_{r}$ goes to zero as well.

\footnotetext{
${ }^{22}$ It is immediate to verify that the condition $0<\underline{B}_{K} \leq \bar{B}_{K}<\infty$ is satisfied as long as the probability $\lambda_{e}$ that an employed worker has the opportunity of searching is not too large.
} 
Lemma 6.4. For any $\rho>0$ and any $J_{n}, J_{r} \in \mathcal{J}$, if $\left\|J_{n}-J_{r}\right\|<\rho$, then

$$
\begin{aligned}
& \left\|K_{n}-K_{r}\right\|<\alpha_{K}(\rho), \\
& \alpha_{K}(\rho) \equiv \beta(1-\delta) \lambda_{e} \bar{K}\left(\bar{B}_{p} \alpha_{h} \rho+\alpha_{p}(\rho)\right) /[1-\beta(1-\delta)] .
\end{aligned}
$$

PROOF: Let $\rho>0$ be an arbitrary real number; let $J_{n}$ and $J_{r}$ be arbitrary functions in $\mathcal{J}$ such that $\left\|J_{n}-J_{r}\right\|<\rho$. Let $(w, y, z)$ be an arbitrary point in $W \times Y \times Z$. The distance between $K_{n}(w, y, z)$ and $K_{r}(w, y, z)$ is such that

$$
\begin{aligned}
& \left|K_{n}(w, y, z)-K_{r}(w, y, z)\right| \\
\leq & \beta(1-\delta) \mathbb{E}_{\hat{s}}\left\{\left|K_{n}(w, y, z)-K_{r}(w, y, z)\right|\right\} \\
+ & \beta(1-\delta) \lambda_{e} \bar{K} \mathbb{E}_{\hat{s}}\left\{\left|\tilde{p}_{n}\left(H_{n}(w, \hat{y}), \hat{y}\right)-\tilde{p}_{n}\left(H_{r}(w, \hat{y}), \hat{y}\right)\right|+\left|\tilde{p}_{n}\left(H_{r}(w, \hat{y}), \hat{y}\right)-\tilde{p}_{r}\left(H_{r}(w, \hat{y}), \hat{y}\right)\right|\right\} \\
< & \beta(1-\delta)\left[\left\|K_{n}-K_{r}\right\|+\lambda_{e} \bar{K}\left(B_{p} \alpha_{h} \rho+\alpha_{p}(\rho)\right)\right],
\end{aligned}
$$

where the last inequality makes use of the bounds in (6.7), (6.9) and (4.4). Since this result holds for all $(w, y, z) \in W \times Y \times Z$, the RHS is an upper bound on $\left\|K_{n}-K_{r}\right\|$. Re-arranging terms yields the bound on $\left\|K_{n}-K_{r}\right\|$ given by $(6.11)$.

\subsection{Existence of a Block Recursive Equilibrium}

In the previous subsections, we have computed the employment value function, $H$, the wage function, $h$, and the firm's value function, $K$, associated with an arbitrary $J \in \mathcal{J}$. In this subsection, we insert $K$ and $h$ into the right hand side of the equilibrium condition (2.6), and we compute an update, $\tilde{J}=T J$, for the value function $J$. More specifically, $\tilde{J}$ is given by

$$
\begin{array}{ll}
\tilde{J}(V, y, z) & =\max _{\pi_{i}, \tilde{V}_{i}} \sum_{i=1}^{2} \pi_{i} K\left(h\left(\tilde{V}_{i}, y\right), y, z\right), \\
\text { s.t. } & V=\sum_{i=1}^{2} \pi_{i} \tilde{V}_{i}, \\
& \pi_{i} \in[0,1], \pi_{1}+\pi_{2}=1, \tilde{V}_{i} \in X .
\end{array}
$$

The updated function, $\tilde{J}$, has four properties. First, $\tilde{J}$ depends on the aggregate state of the economy, $\psi$, only through the aggregate component of productivity, $y$. This property follows immediately from the fact that both the objective function and the choice set on the right hand side of (5.1) depend on $\psi$ only through $y$. Second, the updated value function, $\tilde{J}$, is bi-Lipschitz continuous in $V$. More specifically, for all $(y, z) \in Y \times Z$ and all $V_{1}, V_{2} \in X$, with $V_{1} \leq V_{2}$, the difference $\tilde{J}\left(V_{2}, y, z\right)-\tilde{J}\left(V_{1}, y, z\right)$ is bounded between $-\bar{B}_{K}\left(V_{2}-V_{1}\right)$ and $-\underline{B}_{K}(1-\beta(1-\delta))\left(V_{2}-V_{1}\right)$ (see part (i) in the proof of Lemma 6.5). Third, $\tilde{J}$ is bounded in $[\underline{J}, \bar{J}]$ for some bounds $\underline{J}$ and $\bar{J}$ that are independent of $J$ and $\tilde{J}$. More specifically, for all $(V, y, z) \in X \times Y \times Z, \tilde{J}(V, y, z)$ is greater than $\underline{K}$ and smaller than $\bar{K}$ (see part (ii) in the proof of Lemma 6.5). Finally, $\tilde{J}$ is 
concave in $V$ (see part (iii) in the proof of Lemma 6.5). Therefore, given the appropriate choices of $\underline{B}_{J}, \bar{B}_{J}, \underline{J}$, and $\bar{J}$, the updated value function, $\tilde{J}$, satisfies conditions (J1), (J2) and (J3) and, hence, it belongs to the set $\mathcal{J}$. This argument is formalized in the following lemma:

Lemma 6.5. Set $\underline{J}=\underline{K}$, and $\bar{J}=\bar{K}$. Set $\underline{B}_{J}=\underline{B}_{K}(1-\beta(1-\delta))$ and $\bar{B}_{J}=\bar{B}_{K}$. Then, the updated value function, $\tilde{J}$, belongs to the set $\mathcal{J}$.

Proof: (i) Let $(V, y, z)$ be an arbitrary point in $X \times Y \times Z$. Then, $\tilde{J}(V, y, z)$ is such that

$$
\begin{aligned}
& \tilde{J}(V, y, z) \leq \max _{\tilde{V}_{1} \in X} K\left(h\left(\tilde{V}_{1}, y\right), y, z\right) \leq \max _{w \in W} K(w, y, z) \leq \bar{K}, \\
& \tilde{J}(V, y, z) \geq \min _{\tilde{V}_{1} \in X} K\left(h\left(\tilde{V}_{1}, y\right), y, z\right) \leq \min _{w \in W} K(w, y, z) \geq \underline{K},
\end{aligned}
$$

where we used the fact that if $\tilde{V}_{1} \in X$ then $h\left(\tilde{V}_{1}, y\right) \in W$. The above inequalities imply that $\tilde{J}$ satisfies property $(\mathrm{J} 1)$ of the set $\mathcal{J}$.

(ii) Let $(y, z)$ be an arbitrary point in $Y \times Z$, and $V_{1}, V_{2}$ two arbitrary points in $X$, with $V_{1} \leq V_{2}$. Let $\left\{\pi_{i, 1}, \tilde{V}_{i, 1}\right\}_{i=1}^{2}$ denote the maximizer of (6.12) for $V=V_{1}$, and $\left\{\pi_{i, 2}, \tilde{V}_{i, 2}\right\}_{i=1}^{2}$ the maximizer of $(6.12)$ for $V=V_{2}$. Let $\left\{\Delta_{i, 1}\right\}_{i=1}^{2}$ be a vector such that $\Sigma_{i=1}^{2} \pi_{i, 1}\left(\tilde{V}_{i, 1}+\Delta_{i, 1}\right)=V_{2}$ and $\Delta_{i, 1} \in\left[0, \bar{x}-V_{i, 1}\right]$. Also, let $\left\{\Delta_{i, 2}\right\}_{i=1}^{2}$ be a vector such that $\Sigma_{i=1}^{2} \pi_{i, 2}\left(\tilde{V}_{i, 2}-\Delta_{i, 2}\right)=V_{1}$ and $\Delta_{i, 2} \in\left[0, V_{i, 2}-\underline{x}\right]$. Note that $\left\{\pi_{i, 1}, \tilde{V}_{i, 1}+\Delta_{i, 1}\right\}_{i=1}^{2}$ belongs to the choice set of (6.12) for $V=V_{2}$. Therefore,

$$
\begin{aligned}
\tilde{J}\left(V_{2}, y, z\right)-\tilde{J}\left(V_{1}, y, z\right) & \geq \sum_{i=1}^{2} \pi_{i, 1}\left[K\left(h\left(\tilde{V}_{i, 1}+\Delta_{i, 1}, y\right), y, z\right)-K\left(h\left(\tilde{V}_{i, 1}, y\right), y, z\right)\right] \\
& \geq-\bar{B}_{K}\left[\sum_{i=1}^{2} \pi_{i, 1}\left(h\left(\tilde{V}_{i, 1}+\Delta_{i, 1}, y\right)-h\left(\tilde{V}_{i, 1}, y\right)\right)\right] \\
& =-\bar{B}_{K}\left(V_{2}-V_{1}\right) .
\end{aligned}
$$

Next, note that $\left\{\pi_{i, 2}, \tilde{V}_{i, 2}-\Delta_{i, 2}\right\}_{i=1}^{2}$ belongs to the choice set of (6.12) for $V=V_{2}$. Therefore,

$$
\begin{aligned}
\tilde{J}\left(V_{2}, y, z\right)-\tilde{J}\left(V_{1}, y, z\right) & \leq \sum_{i=1}^{2} \pi_{i, 2}\left[K\left(h\left(\tilde{V}_{i, 2}, y\right), y, z\right)-K\left(h\left(\tilde{V}_{i, 2}-\Delta_{i, 2}, y\right), y, z\right)\right] \\
& \leq-\underline{B}_{K}\left[\sum_{i=1}^{2} \pi_{i, 2}\left(h\left(\tilde{V}_{i, 2}, y\right)-h\left(\tilde{V}_{i, 2}-\Delta_{i, 2}, y\right)\right)\right] \\
& =-\underline{B}_{K}(1-\beta(1-\delta))\left(V_{2}-V_{1}\right) .
\end{aligned}
$$

The above inequalities imply that $\tilde{J}$ satisfies property $(\mathrm{J} 2)$ of the set $\mathcal{J}$.

(iii) Finally, Appendix $\mathrm{F}$ shows that $\tilde{J}$ is concave with respect to $V$. Hence, $\tilde{J}$ satisfies property (J3) of the set $\mathcal{J}$.

Now, consider two arbitrary functions $J_{n}, J_{r} \in \mathcal{J}$. Let $H_{n}, h_{n}, K_{n}$ and $\tilde{J}_{n}$ denote the functions computed with $J_{n}$. Similarly, let $H_{r}, h_{r}, K_{r}$ and $\tilde{J}_{r}$ denote the functions computed with $J_{r} \in \mathcal{J}$. 
Lemma 6.4 proves that as the distance between $J_{n}$ and $J_{r}$ converges to zero, the distance between $\tilde{J}_{n}$ and $\tilde{J}_{r}$ goes to zero as well.

Lemma 6.6. For any $\rho>0$ and any $J_{n}, J_{r} \in \mathcal{J}$, if $\left\|J_{n}-J_{r}\right\|<\rho$, then

$$
\left\|\tilde{J}_{n}-\tilde{J}_{r}\right\|<\alpha_{J}(\rho), \quad \alpha_{J}(\rho) \equiv \alpha_{K}(\rho)+\bar{B}_{K} \alpha_{h} \rho .
$$

PROOF: Let $\rho>0$ be an arbitrary real number; let $J_{n}$ and $J_{r}$ be arbitrary functions in $\mathcal{J}$ such that $\left\|J_{n}-J_{r}\right\|<\rho$. Denote as $H_{n}, h_{n}$ and $K_{n}$ the functions computed with $J_{n}$, and $H_{r} h_{r}$ and $K_{r}$ the functions computed with $J_{r}$. Let $(V, y, z)$ be an arbitrary point in $X \times Y \times Z$. The distance between $\tilde{J}_{n}(V, y, z)$ and $\tilde{J}_{r}(V, y, z)$ is such that

$$
\begin{aligned}
& \left|\tilde{J}_{n}(V, y, z)-\tilde{J}_{r}(V, y, z)\right| \\
\leq & \max _{\tilde{V}_{1} \in X}\left[\left|K_{n}\left(h_{n}\left(\tilde{V}_{1}\right)\right)-K_{n}\left(h_{r}\left(\tilde{V}_{1}\right)\right)\right|+\left|K_{n}\left(h_{r}\left(\tilde{V}_{1}\right)\right)-K_{r}\left(h_{r}\left(\tilde{V}_{1}\right)\right)\right|\right] \\
\leq & \max _{V_{1} \in X}\left[\bar{B}_{K}\left\|h_{n}-h_{r}\right\|+\left\|K_{n}-K_{r}\right\|\right] \leq \alpha_{K}(\rho)+\bar{B}_{K} \alpha_{h} \rho,
\end{aligned}
$$

where the last inequality makes use of the bounds in (6.7), (6.9) and (6.11). Since this result holds for all $(V, y, z) \in X \times Y \times Z$, the RHS is an upper bound on $\left\|\tilde{J}_{n}-\tilde{J}_{r}\right\|$.

Lemma 6.5 implies that the equilibrium operator $T$ maps the set $\mathcal{J}$ into itself. Moreover, since the functions in the set $\mathcal{J}$ are bi-Lipschitz and the sets $Y$ and $Z$ are finite, Lemma 6.5 implies that the family of functions $T(\mathcal{J})$ is equicontinuous. In addition, Lemma 6.6 implies that the operator $T$ is continuous. Since these properties of the operator $T$ are sufficient to apply Schauder's fixed point theorem, there exists a function $J^{*} \in \mathcal{J}$ such that $T J^{*}=J^{*}$. Clearly, the firm's value function $J^{*}$, together with the associated tightness function $\theta^{*}$, search value function $R^{*}$, search policy function $m^{*}$, and unemployment value function $U^{*}$, constitute a Block Recursive Equilibrium. This completes the proof of the following theorem:

Theorem 6.7. There exists a Block Recursive Equilibrium for the proxy of the model with fixed-wage contracts.

For any Block Recursive Equilibrium of the proxy model, we can compute the worker's value of unemployment, $U^{*}(y)$, and the worker's value of employment at the beginning of the search stage, $H^{*}(w, y)+\lambda_{e} \max \left\{0, R^{*}\left(H^{*}(w, y), y\right)\right\}$. A BRE of the proxy model is a BRE of the original model if

$$
U^{*}(y) \leq H^{*}(w, y)+\lambda_{e} \max \left\{0, R^{*}\left(H^{*}(w, y), y\right)\right\}
$$

for all equilibrium wages $w$ and for all realizations of the aggregate component of productivity $y$. This condition implicitly restricts the parameter values of the model. We do not explicitly 
characterize this restriction here. However, notice that, since unemployed workers search for jobs that offer lifetime utility $H^{*}(w, y)$ greater than $U^{*}(y)$ and since employed workers search for even better jobs, (6.14) is likely to be satisfied as long as aggregate productivity shocks are sufficiently small. This is the case in the calibrated example below.

\section{A Calibrated Example}

In Sections 5 and 6, we have established existence of a Block Recursive Equilibrium for our stochastic model of directed search on the job. In this section, we want to illustrate additional properties of a BRE. To this aim, we calibrate the parameters of the model using the data on the US labor market. Given the calibrated parameters, we construct the equilibrium operator $T$ and we apply it to an arbitrary value function, $J \in \mathcal{J}$, until we reach a fixed point, $J^{*}$. Then, we construct a BRE by computing the agents' value functions, policy functions and the market tightness function associated with $J^{*}$. For the sake of brevity, we report our findings only for the version of the model with fixed-wage contracts.

The workers' preferences are described by the discount factor $\beta$, and the value of leisure $b$. The search technology is described by the probability that an unemployed worker is able to look for a job, $\lambda_{u}$, by the probability that an employed worker is able look for a job, $\lambda_{e}$, and by the job-finding probability function, $p(\theta)$. We assume that $p(\theta)$ is of the form $\theta\left(1+\theta^{\gamma}\right)^{-1 / \gamma}$. The production technology is described by the vacancy creation cost $k$, the exogenous job-destruction probability $\delta$, and the stochastic processes for the idiosyncratic and the aggregate components of productivity. We assume that the idiosyncratic component of productivity, $z$, is always equal to zero, and that the aggregate component of productivity, $y$, obeys a two-state Markov process, with $y \in\{0.95,1.05\}$. The unconditional mean of $y$ is normalized to 1 .

We set the model period to be one quarter. We set $\beta$ equal to 0.987 , so that the annual interest rate in the model is 5 percent. We set $k, \delta$, and $\lambda_{e}$ equal to $0.001,0.045$, and 0.3 respectively, so that the average transition rates between employment, unemployment, and across employers are the same in the model as in the US data. ${ }^{23}$ We normalize $\lambda_{u}$ to 1 . We tentatively set $\gamma$ equal to 0.5 , which implies an the elasticity of substitution between vacancies and applicants of $2 / 3$. Finally, we set $b$ equal to 0.7 , so that the consumption value of leisure is 70 percent of the consumption value of work (a figure that is empirically supported by Hall and Milgrom, 2008).

Given these parameter values, we compute a BRE of the proxy model. In Figure 1, we plot the market tightness as a function of the value offered by the firms to the workers, $x$, and conditional

\footnotetext{
${ }^{23}$ The data used for the calibration are described in Section 5 of Menzio and Shi (2008).
} 
on the realization of the aggregate component of productivity, $y$. In accordance with Lemma 4.1, we find that the market tightness is decreasing with respect to $x$. Moreover, we find that the market tightness is higher when the aggregate component of productivity is higher. Intuitively, when $y$ is higher, firms create more vacancies per applicant because the value of filling a vacancy is higher.

In Figure 2, we plot the worker's optimal search strategy as a function of the value, $V$, of his current employment position, and conditional on the realization of the aggregate component of productivity, $y$. In accordance with Lemma 4.3, we find that the worker seeks jobs that offer more generous terms of trade (and are harder to get) when the value of his current employment position is higher. Moreover, we find that the worker seeks jobs that offer more generous terms of trade and are easier to get when the aggregate component of productivity is higher (see Figure $3)$.

In Figure 4, we plot the worker's employment value as a function of the wage $w$, conditional on the realization of the aggregate component of productivity, $y$. Similarly, in Figure 5, we plot the firm's value of employing a worker as a function of the wage $w$, conditional on $y$. We find that the worker's value of being employed is increasing in $w$, while the firm's value of having an employee is decreasing in $w$. Moreover, we find that the worker's and firm's values are both increasing with respect to the the aggregate component of productivity. Intuitively, when $y$ is higher, the worker's value is higher because the value of unemployment and the return to searching are higher. The firm's value is higher because its periodical profit (conditional on the survival of the employment relationship) is higher. From these properties of $H^{*}$ and $K^{*}$, it follows immediately that the firm's value of filling a vacancy in submarket $x$ is decreasing in $x$, and increasing in $y$ (see Figure $6)$.

The equilibrium functions $\theta^{*}, m^{*}$, and $H^{*}$ illustrate that the BRE preserves some attractive qualitative features of the models by Burdett and Mortensen (1998), Postel-Vinay and Robin (2002) and Burdett and Coles (2003). For example, the BRE generates residual wage inequality because, if ex-ante homogeneous workers have different luck with their job searches, they will be employed at different wages. The BRE features a positive return to tenure because workers who are employed at lower wages search in tighter submarkets and, hence, have a higher probability of leaving their job. For the very same reason, the BRE also features a negative relationship between tenure and job hazard.

Finally, using the equilibrium functions $\theta^{*}, m^{*}, H^{*}, K^{*}, J^{*}$ and $U^{*}$, we simulate the life of an unemployed worker. We find that, whenever the worker is employed, he prefers keeping his job 
than moving into unemployment. That is, we find that condition (6.14) is satisfied everywhere along the equilibrium path. Therefore, the BRE of the proxy model is also a BRE of the original model with fixed-wage contracts.

\section{Conclusions}

In this paper, we prove existence of a Block Recursive Equilibrium for a general model of directed search on the job, which allows for aggregate shocks, idiosyncratic shocks, risk aversion, and different specifications of the contractual environment. The BRE of our model preserves a number of attractive qualitative properties of the models of random search on the job by Burdett and Mortensen (1998), Postel-Vinay and Robin (2002), and Burdett and Coles (2003). That is, the BRE features flows of workers between employment, unemployment, and across different employers; it features residual wage inequality, and a positive return to tenure and experience. However, the BRE of our model differs from these models in that it takes into account directed search and that it is tractable for studying dynamics. In the equilibrium of the random search models, the distribution of workers across different employment states is an infinite-dimensional object which non-trivially affects the agents' value and policy functions. In the BRE of our model, the distribution of workers across different employment states does not affect the agents' value and policy functions. For this reason, while solving the equilibrium of the random search model in a stochastic environment is a difficult task both computationally and analytically, solving the BRE of our model is as easy as solving a representative agent model. These properties of the BRE make our model both a useful and a practical tool for studying labor market dynamics.

\section{References}

[1] Acemoglu, D., and R. Shimer (1999): Efficient Unemployment Insurance, Journal of Political Economy, 107, 893-928.

[2] Atkeson, A., and R.E. Lucas, Jr. (1992): On Efficient Distribution with Private Information, Review of Economic Studies, 59, 427-453.

[3] Barlevy, G. (2002): The Sullying Effect of Recessions, Review of Economic Studies, 69, $65-96$.

[4] Burdett, K., and M. Coles (2003): Equilibrium Wage-Tenure Contracts, Econometrica, 71, 1377-1404. 
[5] Burdett, K., and D. Mortensen (1998): Wage Differentials, Employer Size, and Unemployment, International Economic Review, 39, 257-273.

[6] Burdett, K. S. Shi, and R. Wright (2001): Pricing and matching with frictions, Journal of Political Economy, 109, 1060-85.

[7] Delacroix, A., and S. Shi (2006): Directed Search on the Job and the Wage Ladder, International Economic Review, 47, 651-699.

[8] Hall, R., and A.B. Krueger (2008): Wage Formation between newly Hired Workers and Employers: Survey Evidence, manuscript, Stanford University.

[9] Hall, R., and P. Milgrom (2008): The Limited Influence of Unemployment of the Wage Bargain, American Economic Review.

[10] Holzer, H., L. Katz, and A. Krueger (1991): Job Queues and Wages, Quarterly Journal of Economics, 106, 739-68.

[11] Jolivet, G., F. Postel-Vinay, and J. Robin (2006): The Empirical Content of the Job Search Model: Labor Mobility and Wage Distributions in Europe and the US, European Economic Review, 50, 877-907.

[12] Menzio, G. (2007): A Theory of Partially Directed Search, Journal of Political Economy, $115,748-769$.

[13] Menzio, G., and S. Shi (2008): Efficient Search On the Job and the Business Cycle, Working Paper 308, University of Toronto.

[14] Milgrom, P., and I. Segal (2002): Envelope Theorems for Arbitrary Choice Sets, Econometrica, 70, 583-601.

[15] Moen, E. (1997): Competitive Search Equilibrium, Journal of Political Economy, 105, 694723.

[16] Moen, E., and A. Rosen (2004): Does Poaching Distort Training? Rev. Econ. Studies 71: $1143-62$.

[17] Montgomery, J. (1991): Equilibrium Wage Dispersion and Interindustry Wage Differentials, Quarterly Journal of Economics, 106, 163-79.

[18] Mortensen, D. (1994): The Cyclical Behavior of Job and Worker Flows, Journal of Economic Dynamics and Control, 18, 1121-1142. 
[19] Moscarini, G., and F. Postel-Vinay (2008): Non Stationary Search Equilibrium. Manuscript, Yale University.

[20] Peters, M. (1991): Ex Ante Price Offers in Matching Games: Non-Steady State, Econometrica, 59, 1425-1454.

[21] Pissarides, C. (1994): Search Unemployment with On-the-job Search, Review of Economic Studies, 61, 457-475.

[22] Postel-Vinay, F., and J. Robin (2002): Equilibrium Wage Dispersion with Worker and Employer Heterogeneity, Econometrica, 70, 2295-2350.

[23] Prescott, E.C., and R.M. Townsend (1984): Pareto Optima and Competitive Equilibria with Adverse Selection and Moral Hazard, Econometrica, 52, 21-46.

[24] Royden, H. (1988): Real Analysis. Upper Saddle River, New Jersey: Prentice-Hall.

[25] Shi, S. (2009): Directed Search for Equilibrium Wage-Tenure Contracts, Econometrica, 77, 561-584.

[26] Shimer, R. (2005): The Cyclical Behavior of Unemployment and Vacancies, American Economic Review, 95, 25-49.

[27] Stokey, N., R.E. Lucas, with E. Prescott (1989): Recursive Methods in Economic Dynamics. Cambridge, Massachusetts: Harvard University Press. 


\section{Appendix}

\section{A. Properties of the Set of Functions $\mathcal{J}$}

Lemma A.1. $\mathcal{J}$ is a non-empty, bounded, closed and convex subset of the space of bounded, continuous functions on $X \times Y \times Z$, with the sup norm.

Proof: (i) Clearly, the set $\mathcal{J}$ is non-empty and bounded.

(ii) Next, we need to prove that the set $\mathcal{J}$ is closed. To this aim, let $\left\{J_{n}\right\}_{n=1}^{\infty}$ be an arbitrary sequence with $J_{n} \in \mathcal{J}$ for every $n$, and with $J_{n} \rightarrow J$ (in the sup norm). Note that, since $J_{n} \rightarrow J$, for every $\varepsilon>0$, there exists $N(\varepsilon) \geq 1$ such that $n \geq N(\varepsilon) \Longrightarrow\left\|J_{n}-J\right\|<\varepsilon$.

For arbitrary $(y, z) \in Y \times Z$ and arbitrary $V_{1}, V_{2} \in X$, with $V_{1} \leq V_{2}$, suppose that the difference $J\left(V_{2}, y, z\right)-J\left(V_{1}, y, z\right)$ is strictly smaller than $-\bar{B}_{J}\left(V_{2}-V_{1}\right)$. Let $\varepsilon>0$ be one third of the difference between $-\bar{B}_{J}\left(V_{2}-V_{1}\right)$ and $\left[J\left(V_{2}, y, z\right)-J\left(V_{1}, y, z\right)\right]$. Let $n$ be a natural number greater than $N(\varepsilon)$. Since $\left\|J_{n}-J\right\|<\varepsilon$, the difference $J_{n}\left(V_{2}, y, z\right)-J_{n}\left(V_{1}, y, z\right)$ is such that

$$
\begin{aligned}
J_{n}\left(V_{2}, y, z\right)-J_{n}\left(V_{1}, y, z\right) & <J\left(V_{2}, y, z\right)-J\left(V_{1}, y, z\right)+2 \varepsilon \\
& =\frac{1}{3}\left[J\left(V_{2}, y, z\right)-J\left(V_{1}, y, z\right)\right]-\frac{2}{3} \bar{B}_{J}\left(V_{2}-V_{1}\right) \\
& <-\bar{B}_{J}\left(V_{2}-V_{1}\right) .
\end{aligned}
$$

The last inequality contradicts $J_{n} \in \mathcal{J}$. Therefore, $J\left(V_{2}, y, z\right)-J\left(V_{1}, y, z\right)$ is greater than $-\bar{B}_{J}\left(V_{2}-V_{1}\right)$ for all $(y, z) \in Y \times Z$ and all $V_{1}, V_{2} \in X$, with $V_{1} \leq V_{2}$. Using a similar argument, we can prove that $J\left(V_{2}, y, z\right)-J\left(V_{1}, y, z\right)$ is smaller than $-\underline{B}_{J}\left(V_{2}-V_{1}\right)$ for all $(y, z) \in Y \times Z$ and all $V_{1}, V_{2} \in X$, with $V_{1} \leq V_{2}$. That is, $J$ satisfies property (J1) of the set $\mathcal{J}$.

For all $(V, y, z) \in X \times Y \times Z$, it is immediate to verify that $J(V, y, z) \in[\underline{J}, \bar{J}]$. Hence, $J$ satisfies property $(\mathrm{J} 2)$ of the set $\mathcal{J}$. For arbitrary $(y, z) \in Y \times Z$, arbitrary $V_{1}, V_{2} \in X$, and arbitrary $\alpha \in$ $[0,1]$, suppose that $J\left(V_{\alpha}, y, z\right)$ is strictly smaller than $\alpha J\left(V_{1}, y, z\right)+(1-\alpha) J\left(V_{2}, y, z\right)$, where $V_{\alpha}=$ $\alpha V_{1}+(1-\alpha) V_{2}$. Let $\varepsilon>0$ be one third of the difference between $\left[\alpha J\left(V_{1}, y, z\right)+(1-\alpha) J\left(V_{2}, y, z\right)\right]$ and $J\left(V_{\alpha}, y, z\right) . n$ be a natural number greater than $N(\varepsilon)$. Since $\left\|J_{n}-J\right\|<\varepsilon$, we have

$$
\begin{aligned}
J_{n}\left(V_{\alpha}, y, z\right) & <J\left(V_{\alpha}, y, z\right)+\varepsilon \\
& =\alpha J\left(V_{1}, y, z\right)+(1-\alpha) J\left(V_{2}, y, z\right)-2 \varepsilon \\
& <\alpha J_{n}\left(V_{1}, y, z\right)+(1-\alpha) J_{n}\left(V_{2}, y, z\right)-\varepsilon \\
& <\alpha J_{n}\left(V_{1}, y, z\right)+(1-\alpha) J_{n}\left(V_{2}, y, z\right) .
\end{aligned}
$$

The last inequality contradicts $J_{n} \in \mathcal{J}$. Therefore, $J\left(V_{\alpha}, y, z\right)$ is greater than $\alpha J\left(V_{1}, y, z\right)+(1-$ $\alpha) J\left(V_{2}, y, z\right)$ for all $(y, z) \in Y \times Z$, all $V_{1}, V_{2} \in X$ and all $\alpha \in[0,1]$. That is, $J$ satisfies property (J3) of the set $\mathcal{J}$. This establishes that $J \in \mathcal{J}$ and, hence, that the set $\mathcal{J}$ is closed. 
(iii) Finally, we need to prove that the set $\mathcal{J}$ is convex. To this aim, consider arbitrary $J_{1}$, $J_{2} \in \mathcal{J}$ and an arbitrary $\alpha \in[0,1]$. Denote $J_{\alpha}(V, y, z)=\alpha J_{1}(V, y, z)+(1-\alpha) J_{2}(V, y, z)$. For all $(y, z) \in Y \times Z$ and all $V_{1}, V_{2} \in X$, with $V_{1} \leq V_{2}$, the difference $J_{\alpha}\left(V_{2}, y, z\right)-J_{\alpha}\left(V_{1}, y, z\right)$ is such that

$$
\begin{aligned}
& J_{\alpha}\left(V_{2}, y, z\right)-J_{\alpha}\left(V_{1}, y, z\right) \\
= & \alpha\left[J_{1}\left(V_{2}, y, z\right)-J_{1}\left(V_{1}, y, z\right)\right]+(1-\alpha)\left[J_{2}\left(V_{2}, y, z\right)-J_{2}\left(V_{1}, y, z\right)\right] \\
\in \quad & {\left[-\underline{B}_{J}\left(V_{2}-V_{1}\right),-\bar{B}_{J}\left(V_{2}-V_{1}\right)\right] . }
\end{aligned}
$$

Hence, $J_{\alpha}$ satisfies property $(\mathrm{J} 1)$ of the set $\mathcal{J}$. For all $(V, y, z) \in X \times Y \times Z$, it is immediate to verify that $J_{\alpha}(V, y, z) \in[\underline{J}, \bar{J}]$. Hence, $J_{\alpha}$ satisfies property $(\mathrm{J} 2)$ of the set $\mathcal{J}$. For all $(y, z) \in Y \times Z$, $V_{1}, V_{2} \in X$, and $\zeta \in[0,1]$, let $V_{\zeta}=\zeta V_{1}+(1-\zeta) V_{2}$. Then, $J_{\alpha}\left(V_{\zeta}, y, z\right)$ is such that

$$
\begin{aligned}
& J_{\alpha}\left(V_{\zeta}, y, z\right) \\
= & \alpha J_{1}\left(V_{\zeta}, y, z\right)+(1-\alpha) J_{2}\left(V_{\zeta}, y, z\right) \\
\geq & \alpha\left[\zeta J_{1}\left(V_{1}, y, z\right)+(1-\zeta) J_{1}\left(V_{2}, y, z\right)\right]+(1-\alpha)\left[\zeta J_{2}\left(V_{1}, y, z\right)+(1-\zeta) J_{2}\left(V_{2}, y, z\right)\right] \\
= & \zeta J_{\alpha}\left(V_{1}, y, z\right)+(1-\zeta) J_{\alpha}\left(V_{2}, y, z\right) .
\end{aligned}
$$

Hence, $J_{\alpha}$ satisfies property $(\mathrm{J} 3)$ of the set $\mathcal{J}$. This establishes that $J_{\alpha} \in \mathcal{J}$ and, hence, that the set $\mathcal{J}$ is convex.

\section{B. Proof of Lemma 4.1}

(i) For the sake of brevity, let us suppress the dependence of various functions on $y$ and $z$. Let $y$ be an arbitrary point in $Y$, and let $x_{1}, x_{2}$ be two points in $X$ with $x_{1} \leq x_{2}$. First, consider the case in which $x_{1} \leq x_{2} \leq \tilde{x}$. In this case, the difference $\theta\left(x_{2}\right)-\theta\left(x_{1}\right)$ is equal to

$$
\theta\left(x_{2}\right)-\theta\left(x_{1}\right)=q^{-1}\left(k / J\left(x_{2}\right)\right)-q^{-1}\left(k / J\left(x_{1}\right)\right)=\int_{k / J\left(x_{1}\right)}^{k / J\left(x_{2}\right)} q^{-1 \prime}(t) d t,
$$

where the first equality makes use of (4.1), and the second equality makes use of the fact that $J\left(x_{1}\right) \geq J\left(x_{2}\right) \geq k>0$. For all $x \in[\underline{x}, \tilde{x}]$, the derivative of the inverse function $q^{-1}($.$) evaluated$ at $k / J(x)$ is equal to $1 / q^{\prime}(\theta(x)) \in\left[1 / q^{\prime}(\bar{\theta}), 1 / q^{\prime}(0)\right]$, where $1 / q^{\prime}(\bar{\theta}) \leq 1 / q^{\prime}(0)<0$. Therefore, the last term in (B.1) is such that

$$
\frac{1}{q^{\prime}(\bar{\theta})}\left(\frac{k}{J\left(x_{2}\right)}-\frac{k}{J\left(x_{1}\right)}\right) \leq \int_{k / J\left(x_{1}\right)}^{k / J\left(x_{2}\right)} q^{-1 \prime}(t) d t \leq \frac{1}{q^{\prime}(0)}\left(\frac{k}{J\left(x_{2}\right)}-\frac{k}{J\left(x_{1}\right)}\right) .
$$

The difference $k / J\left(x_{2}\right)-k / J\left(x_{1}\right)$ is equal to

$$
\frac{k}{J\left(x_{2}\right)}-\frac{k}{J\left(x_{1}\right)}=\int_{J\left(x_{2}\right)}^{J\left(x_{1}\right)} \frac{k}{t^{2}} d t
$$


For all $x \in[\underline{x}, \tilde{x}], J(x)$ is strictly decreasing in $x$ and it is bounded between $\bar{J}$ and $k$. Therefore, the integral on the RHS above is such that

$$
\begin{aligned}
& \int_{J\left(x_{2}\right)}^{J\left(x_{1}\right)} \frac{k}{t^{2}} d t \leq \frac{1}{k}\left[J\left(x_{1}\right)-J\left(x_{2}\right)\right] \leq \frac{\bar{B}_{J}}{k}\left(x_{2}-x_{1}\right), \\
& \int_{J\left(x_{2}\right)}^{J\left(x_{1}\right)} \frac{k}{t^{2}} d t \geq \frac{k}{\bar{J}^{2}}\left[J\left(x_{1}\right)-J\left(x_{2}\right)\right] \geq \frac{\underline{B}_{J} k}{\bar{J}^{2}}\left(x_{2}-x_{1}\right) .
\end{aligned}
$$

Taken together, (B.2) and (B.3) imply that the difference $\theta\left(x_{2}\right)-\theta\left(x_{1}\right)$ is such that

$$
\frac{\bar{B}_{J}}{q^{\prime}(\bar{\theta}) k}\left(x_{2}-x_{1}\right) \leq \theta\left(x_{2}\right)-\theta\left(x_{1}\right) \leq \frac{\underline{B}_{J} k}{q^{\prime}(0) \bar{J}^{2}}\left(x_{2}-x_{1}\right) .
$$

Next, consider the case in which $x_{1} \leq \tilde{x} \leq x_{2}$. In this case, the difference $\theta\left(x_{2}\right)-\theta\left(x_{1}\right)$ is such that

$$
\begin{aligned}
& \theta\left(x_{2}\right)-\theta\left(x_{1}\right)=\theta(\tilde{x})-\theta\left(x_{1}\right) \leq \frac{\underline{B}_{J} k}{q^{\prime}(0) \bar{J}^{2}}\left(\tilde{x}-x_{1}\right) \leq 0, \\
& \theta\left(x_{2}\right)-\theta\left(x_{1}\right)=\theta(\tilde{x})-\theta\left(x_{1}\right) \geq \frac{\bar{B}_{J}}{q^{\prime}(\bar{\theta}) k}\left(\tilde{x}-x_{1}\right) \geq \frac{\bar{B}_{J}}{q^{\prime}(\bar{\theta}) k}\left(x_{2}-x_{1}\right),
\end{aligned}
$$

where the first equality in both lines makes use of the fact that $\theta\left(x_{2}\right)=\theta(\tilde{x})$, and the first inequality in both lines makes use of the bounds in (B.4). Finally, consider the case in which $\tilde{x} \leq x_{1} \leq x_{2}$. In this case, (4.1) implies that $\theta\left(x_{1}\right)=\theta\left(x_{2}\right)=0$.

(ii) The function $p(\theta)$ is strictly increasing in $\theta$. The function $\theta(x)$ is strictly decreasing in $x$ for all $x \in[\underline{x}, \tilde{x}]$. Therefore, the composite function $p(\theta(x))$ is strictly decreasing in $x$ for $x \in[\underline{x}, \tilde{x}]$. In order to prove that the composite function $p(\theta(x))$ is strictly concave in $x$ for $x \in[\underline{x}, \tilde{x}]$, consider arbitrary $x_{1}, x_{2} \in[\underline{x}, \tilde{x}]$, with $x_{1} \neq x_{2}$, and an arbitrary number $\alpha \in(0,1)$. Let $x_{\alpha}=\alpha x_{1}+(1-\alpha) x_{2}$. Since the function $J(x)$ is concave in $x$ and the function $k / x$ is strictly convex in $x$, we have

$$
\frac{k}{J\left(x_{\alpha}\right)} \leq \frac{k}{\alpha J\left(x_{1}\right)+(1-\alpha) J\left(x_{2}\right)}<\alpha \frac{k}{J\left(x_{1}\right)}+(1-\alpha) \frac{k}{J\left(x_{2}\right)} .
$$

Since $p\left(q^{-1}().\right)$ is strictly decreasing and weakly concave, the previous inequality implies that

$$
\begin{aligned}
p\left(q^{-1}\left(k / J\left(x_{\alpha}\right)\right)\right. & >p\left(q^{-1}\left(\alpha k / J\left(x_{1}\right)+(1-\alpha) k / J\left(x_{2}\right)\right)\right) \\
& \geq \alpha p\left(q^{-1}\left(k / J\left(x_{1}\right)\right)\right)+(1-\alpha) p\left(q^{-1}\left(k / J\left(x_{2}\right)\right)\right) .
\end{aligned}
$$

Since $q^{-1}(k / J(x))$ is equal to $\theta(x)$ for all $x \in[\underline{x}, \tilde{x}]$, (B.5) can be rewritten as

$$
p\left(\theta\left(x_{\alpha}\right)\right)>\alpha p\left(\theta\left(x_{1}\right)\right)+(1-\alpha) p\left(\theta\left(x_{2}\right)\right) .
$$

This establishes that the composite function $p(\theta(x))$ is strictly concave in $x$ for all $x \in[\underline{x}, \tilde{x}]$. 


\section{Proof of Lemma 4.6}

(i) For all $\varphi_{1}, \varphi_{2} \in \mathcal{C}(Y)$, with $\varphi_{1} \leq \varphi_{2}$, the difference $T_{U} \varphi_{2}-T_{U} \varphi_{1}$ is such that

$$
\begin{aligned}
& \left(T_{U} \varphi_{2}\right)(y)-\left(T_{U} \varphi_{1}\right)(y) \\
= & \beta \mathbb{E}_{\hat{\psi}}\left\{\varphi_{2}(\hat{y})-\varphi_{1}(\hat{y})+\lambda_{u}\left[\max \left\{0, R\left(\varphi_{2}(\hat{y}), \hat{y}\right)\right\}-\max \left\{0, R\left(\varphi_{1}(\hat{y}), \hat{y}\right)\right\}\right]\right\} \geq 0,
\end{aligned}
$$

where the last inequality uses the fact that the function $V+\lambda_{u} \max \{0, R(V)\}$ is increasing in $V$. For all $\varphi \in \mathcal{C}(Y)$ and all $a \geq 0, T_{U}(\varphi+a)$ is such that

$$
\begin{aligned}
{\left[T_{U}(\varphi+a)\right](y) } & =\left(T_{U} \varphi\right)(y)+\beta \mathbb{E}_{\hat{\psi}}\left\{a+\lambda_{u}[\max \{R(\varphi+a), 0\}-\max \{R(\varphi), 0\}]\right\} \\
& \leq\left(T_{U} \varphi\right)(y)+\beta a,
\end{aligned}
$$

where, for the sake of brevity, we have suppressed the dependence of various variables from the aggregate state $\hat{y}$. Conditions (C.1) and (C.2) are sufficient to prove that the operator $T_{U}$ is a contraction mapping (Stokey and Lucas with Prescott, 1989, Theorem 3.3). Hence, there exists one and only one $U$ such that $T_{U} U=U$.

(ii) Let $\varphi \in \mathcal{C}(Y)$ be a function that is bounded between $\underline{U}$ and $\bar{U}$. Then, $T_{U} \varphi$ is such that

$$
\begin{aligned}
& \left(T_{U} \varphi\right)(y) \geq v(b)+\beta \underline{U}=\underline{U}, \\
& \left(T_{U} \varphi\right)(y) \leq v(b)+\beta \bar{x}=\bar{U},
\end{aligned}
$$

where the first line makes use of the facts that $\varphi \geq \underline{U}$ and $R(\varphi(\hat{y}), \hat{y}) \geq 0$; and the second line makes use of the fact that $\varphi+\lambda_{u} \max \{0, R(\varphi(\hat{y}), \hat{y})\} \leq \bar{x}$. From the inequalities in (C.3), it follows that the operator $T_{U}$ maps the set of functions that are bounded between $\underline{U}$ and $\bar{U}$ into itself. Since the operator $T_{U}$ is a contraction, it follows that its fixed point, $U$, is bounded between $\underline{U}$ and $\bar{U}$.

\section{Proof of Lemma 6.1}

(i) For all $\varphi_{1}, \varphi_{2} \in \mathcal{C}(W \times Y)$, with $\varphi_{1} \leq \varphi_{2}$, the difference $T_{H} \varphi_{2}-T_{H} \varphi_{1}$ is such that

$$
\begin{aligned}
& \left(T_{H} \varphi_{2}\right)(w, y)-\left(T_{H} \varphi_{1}\right)(w, y) \\
& =\beta(1-\delta) \mathbb{E}_{\hat{y}}\left[\begin{array}{l}
\varphi_{2}(w, \hat{y})+\lambda_{e} \max \left\{0, R\left(\varphi_{2}(w, \hat{y}), \hat{y}\right)\right\} \\
-\varphi_{1}(w, \hat{y})-\lambda_{e} \max \left\{0, R\left(\varphi_{1}(w, \hat{y}), \hat{y}\right)\right\}
\end{array}\right] \geq 0
\end{aligned}
$$

where the last inequality uses the fact that the function $V+\lambda_{e} \max \{0, R(V, \hat{y})\}$ is increasing in $V$. For all $\varphi \in \mathcal{C}(W \times Y)$ and all $a \geq 0, T_{H}(\varphi+a)$ is such that

$$
\begin{aligned}
{\left[T_{H}(\varphi+a)\right](w, y) } & =w+\beta \mathbb{E}_{\hat{y}}\left\{\delta U+(1-\delta)\left[\varphi(w, \hat{y})+\lambda_{e} \max \{0, R(\varphi(w, \hat{y}), \hat{y})\}\right]\right\} \\
& +\beta(1-\delta) \mathbb{E}_{\hat{y}}\left\{a+\lambda_{e} \max \{0, R(\varphi(w, \hat{y}), \hat{y})\}-\lambda_{e} \max \{0, R(\varphi(w, \hat{y})+a, \hat{y})\}\right\} \\
& \leq\left(T_{H} \varphi\right)(w, y)+\beta(1-\delta) a,
\end{aligned}
$$


where the last inequality uses the fact that $R(V, \hat{y})-R(V+a, \hat{y}) \leq 0$. Conditions (D.1) and (D.2) are sufficient to prove that the operator $T_{H}$ is a contraction mapping. Hence, there exists one and only one $H$ such that $T_{H} H=H$.

(ii) Let $\varphi \in \mathcal{C}(W \times Y)$ be a function that satisfies condition (6.4). Let $y$ be an arbitrary point in $Y$, and $w_{1}, w_{2}$ two arbitrary points in $W$ with $w_{1} \leq w_{2}$. For all $\hat{y} \in Y$, the difference $f\left(w_{2}, \hat{y}\right)-f\left(w_{1}, \hat{y}\right)$ is bounded between 0 and $[1-\beta(1-\delta)]^{-1}\left(w_{2}-w_{1}\right)$. Therefore,

$$
\begin{aligned}
& \left(T_{H} \varphi\right)\left(w_{2}, y\right)-\left(T_{H} \varphi\right)\left(w_{1}, y\right) \\
& =w_{2}-w_{1}+\beta(1-\delta) \mathbb{E}_{\hat{y}}\left[\begin{array}{l}
\varphi\left(w_{2}, \hat{y}\right)+\lambda_{e} \max \left\{0, R\left(\varphi\left(w_{2}, \hat{y}\right), \hat{y}\right)\right\} \\
-\varphi\left(w_{1}, \hat{y}\right)-\lambda_{e} \max \left\{0, R\left(\varphi\left(w_{1}, \hat{y}\right), \hat{y}\right)\right\}
\end{array}\right] \\
& \in[1,1 /[1-\beta(1-\delta)]]\left(w_{2}-w_{1}\right) .
\end{aligned}
$$

The bounds in (D.3) imply that the operator $T_{H}$ maps functions that satisfy (6.4) into functions that satisfy (6.4). Since $T_{H}$ is a contraction, its unique fixed point $H$ satisfies (6.4).

(iii) Let $\bar{V}$ denote $(\bar{w}+\beta \delta \bar{U}) /[1-\beta(1-\delta)]$. Let $\varphi \in \mathcal{C}(W \times Y)$ be an arbitrary function such that $\left(T_{H} f\right)(\bar{w}, y) \in[\bar{x}, \bar{V}]$ for all $y \in Y$. The function $T_{H} \varphi$ is such that $\left(T_{H} \varphi\right)(\bar{w}, y) \in[\bar{x}, \bar{V}]$ for all $y \in Y$, because

$$
\begin{aligned}
& \left(T_{H} \varphi\right)(\bar{w}, y) \geq \bar{w}+\beta \delta \underline{U}+\beta(1-\delta) \bar{x}=\bar{x}, \\
& \left(T_{H} \varphi\right)(\bar{w}, y) \leq \bar{w}+\beta \delta \bar{U}+\beta(1-\delta) \bar{V}=\bar{V} .
\end{aligned}
$$

Therefore, the fixed point, $H$, is such that $H(\bar{w}, y) \in[\bar{x}, \bar{V}]$ for all $y \in Y$. Moreover, $H(\underline{w}, y) \leq \underline{x}$ for all $y \in Y$, because

$$
H(\underline{w}, y) \leq \underline{w}+\beta\left[\delta \bar{U}+(1-\delta) \mathbb{E}_{\hat{y}}\left[\bar{V}+\lambda_{e} \max \{0, R(\bar{V}, \hat{y})\}\right]\right] \leq \underline{x} .
$$

\section{E. Proof of Lemma 6.3}

(i) It is immediate to verify that, for all $\varphi_{1}, \varphi_{2} \in \mathcal{C}(W \times Y \times Z)$, if $\varphi_{1} \leq \varphi_{2}$ then $T_{K} \varphi_{1} \leq T_{K} \varphi_{2}$. It is also immediate to verify that, for all $\varphi \in \mathcal{C}(W \times Y \times Z)$ and all $a>0, T_{K}(\varphi+a)$ is smaller than $T_{K} \varphi+\beta a$. These two conditions are sufficient to prove that the operator $T_{K}$ is a contraction mapping. Hence, there exists one and only one $K \in \mathcal{C}(W \times Y \times Z)$ such that $T_{K} K=K$.

(ii)-(iii) Let $\varphi \in \mathcal{C}(W \times Y \times Z)$ satisfy conditions (6.9)-(6.10). Let $(y, z)$ be an arbitrary point in $Y \times Z$, and $w_{1}, w_{2}$ arbitrary points in $W$ with $w_{1} \leq w_{2}$. The difference between $\left(T_{K} \varphi\right)\left(w_{2}, y, z\right)$ 
and $\left(T_{K} \varphi\right)\left(w_{1}, y, z\right)$ is such that

$$
\begin{aligned}
& \left(T_{K} \varphi\right)\left(w_{2}, y, z\right)-\left(T_{K} \varphi\right)\left(w_{1}, y, z\right) \\
= & w_{1}-w_{2}+\beta(1-\delta) \mathbb{E}_{\hat{s}}\left\{\left[1-\lambda_{e} \tilde{p}\left(H\left(w_{2}, \hat{y}\right), \hat{y}\right)\right]\left[\varphi\left(w_{2}, \hat{y}, \hat{z}\right)-\varphi\left(w_{1}, \hat{y}, \hat{z}\right)\right]\right\} \\
+ & \beta(1-\delta) \mathbb{E}_{\hat{s}}\left\{\lambda_{e}\left[\tilde{p}\left(H\left(w_{1}, \hat{y}\right), \hat{y}\right)-\tilde{p}\left(H\left(w_{2}, \hat{y}\right), \hat{y}\right)\right] \varphi\left(w_{1}, \hat{y}, \hat{z}\right)\right\} \\
\leq & -\left\{1+\beta(1-\delta)\left(1-\lambda_{e}\right) \underline{B}_{K}-[1-\beta(1-\delta)]^{-1} \beta(1-\delta) \lambda_{e} \bar{B}_{p} \bar{K}\right\}\left(w_{2}-w_{1}\right) \\
= & -\underline{B}_{K}\left(w_{2}-w_{1}\right),
\end{aligned}
$$

where the first inequality makes use of the bounds in (6.6), (6.9), (4.8) and (6.10). Moreover, the difference between $\left(T_{K} \varphi\right)\left(w_{2}, y, z\right)$ and $\left(T_{K} \varphi\right)\left(w_{1}, y, z\right)$ is such that

$$
\begin{aligned}
& \left(T_{K} \varphi\right)\left(w_{2}, y, z\right)-\left(T_{K} \varphi\right)\left(w_{1}, y, z\right) \\
\geq & -\left\{1+\beta(1-\delta) \bar{B}_{K}-[1-\beta(1-\delta)]^{-1} \beta(1-\delta) \lambda_{e} \bar{B}_{p} \underline{K}\right\}\left(w_{2}-w_{1}\right) \\
= & -\bar{B}_{K}\left(w_{2}-w_{1}\right),
\end{aligned}
$$

where the first inequality makes use of the bounds (6.6), (6.9), (4.8) and (6.10).

Let $w$ be an arbitrary point in $W$. Then, $T_{K} \varphi$ is such that

$$
\begin{aligned}
& \left(T_{K} \varphi\right)(w, y, z) \leq \bar{y}+\bar{z}-\underline{w}+\beta(1-\delta) \bar{K} \leq \bar{K}, \\
& \left(T_{K} \varphi\right)(w, y, z) \geq \underline{y}+\underline{z}-\bar{w}+\beta(1-\delta)\left(1-\lambda_{e}\right) \underline{K} \geq \underline{K} .
\end{aligned}
$$

Inequalities (E.1)-(E.3) imply that the operator $T_{K}$ maps functions that satisfy conditions (6.9)(6.10) into functions that satisfy (6.9)-(6.10). Since the operator $T_{K}$ is a contraction, its unique fixed point, $K$, satisfies conditions (6.9)-(6.10).

\section{F. Two-Point Lotteries and Concavity of the Value Function}

Let $K(x)$ be a continuous function, where $x \in[\underline{x}, \bar{x}]$. Consider the following problem with a two-point lottery:

$$
\begin{array}{ll}
J(V)=\max _{\left(\pi, x_{1}, x_{2}\right)}\left[\pi K\left(x_{1}\right)+(1-\pi) K\left(x_{2}\right)\right] \\
\text { s.t. } \quad \pi x_{1}+(1-\pi) x_{2}=V, \quad x_{1} \leq V \leq x_{2}, \quad \pi \in[0,1] .
\end{array}
$$

The above problem encompasses the maximization problems in (5.1) and (6.12) as special cases. (In these problems, the lottery is contingent on the realizations of aggregate and match-specific shocks, $(y, z)$, which is suppressed here.)

We want to prove that $J(V)$ is concave. To this end, consider arbitrary $V \in(\underline{x}, \bar{x})$. Let $\left(x_{1}^{*}, x_{2}^{*}\right)$ be the solution for $\left(x_{1}, x_{2}\right)$ in (F.1). If $K(V)$ is strictly convex at $V$, it must be true that 
$x_{1}^{*}<V<x_{2}^{*}$. Thus, if $x_{1}^{*}=x_{2}^{*}$, then $J(V)=K(V)$ must be concave at $V$. In the remainder of the proof, it suffices to examine the case where $x_{1}^{*}<x_{2}^{*}$. For any arbitrary $x_{1}, x_{2} \in(\underline{x}, \bar{x})$, $x_{1}<x_{2}$, denote the line segment connecting $K\left(x_{1}\right)$ and $K\left(x_{2}\right)$ as $\overline{x_{1} x_{2}}$, and denote the slope of $\overline{x_{1} x_{2}}$ as

$$
L\left(x_{1}, x_{2}\right) \equiv \frac{K\left(x_{2}\right)-K\left(x_{1}\right)}{x_{2}-x_{1}} .
$$

Using the constraint in (F.1) to express $\pi=\left(x_{2}-V\right) /\left(x_{2}-x_{1}\right)$, we can rewrite $J(V)$ in the following equivalent forms:

$$
J(V)=\max _{\left(x_{1}, x_{2}\right)}\left[K\left(x_{2}\right)-\left(x_{2}-V\right) L\left(x_{1}, x_{2}\right)\right]=\max _{\left(x_{1}, x_{2}\right)}\left[K\left(x_{1}\right)+\left(V-x_{1}\right) L\left(x_{1}, x_{2}\right)\right] .
$$

The following results hold:

(A) For all $x \in[\underline{x}, \bar{x}], K(x)$ must lie on or below the extension of $\overline{x_{1}^{*} x_{2}^{*}}$, i.e., $K(x) \leq K\left(x_{1}^{*}\right)+$ $L\left(x_{1}^{*}, x_{2}^{*}\right)\left(x-x_{1}^{*}\right)$

(B) If $x_{2}^{*}>V$, then $x_{1}^{*}=\arg \min _{x \leq x_{2}^{*}} L\left(x, x_{2}^{*}\right)$ and $x_{2}^{*}=\arg \max _{x \geq x_{1}^{*}} L\left(x_{1}^{*}, x\right)$.

Proofs of (A) AND (B). For (A), consider first the case $x \in\left[x_{1}^{*}, x_{2}^{*}\right]$. (We will return to the case $x \notin\left[x_{1}^{*}, x_{2}^{*}\right]$ after proving (B).) Result (A) holds trivially when $x=x_{1}^{*}$ or $x=x_{2}^{*}$. To show that (A) also holds for $x \in\left(x_{1}^{*}, x_{2}^{*}\right)$, suppose to the contrary that (A) is violated by some $x_{0} \in\left(x_{1}^{*}, x_{2}^{*}\right)$. Then, $K\left(x_{0}\right)>K\left(x_{1}^{*}\right)+L\left(x_{1}^{*}, x_{2}^{*}\right)\left(x_{0}-x_{1}^{*}\right)$. If $x_{0}=V$, then $\left(x_{0}, x_{0}\right)$ is optimal. If $x_{0}<V$, then $\left(x_{0}, x_{2}^{*}\right)$ is feasible and dominates $\left(x_{1}^{*}, x_{2}^{*}\right)$. If $x_{0}>V$, then $\left(x_{1}^{*}, x_{0}\right)$ is feasible and dominates $\left(x_{1}^{*}, x_{2}^{*}\right)$. The result in each of these cases contradicts the optimality of $\left(x_{1}^{*}, x_{2}^{*}\right)$.

For (B), we only prove the first part, i.e., the part for $x_{1}^{*}$, since the proof of the result for $x_{2}^{*}$ is similar. From the first rewritten form of the maximization problem, $L\left(x_{1}^{*}, x_{2}^{*}\right) \leq L\left(x, x_{2}^{*}\right)$ for all $x \leq V$. For $x \in\left(V, x_{2}^{*}\right), K(x)$ is on or below the line connecting $K\left(x_{1}^{*}\right)$ and $K\left(x_{2}^{*}\right)$ (see the proven part of (A) above), and so $L\left(x_{1}^{*}, x_{2}^{*}\right) \leq L\left(x, x_{2}^{*}\right)$. Thus, (B) holds.

Now we prove that $(\mathrm{A})$ also holds for $x \notin\left[x_{1}^{*}, x_{2}^{*}\right]$. If (A) did not hold for some $x_{0}<x_{1}^{*}$, then $L\left(x_{0}, x_{2}^{*}\right)<L\left(x_{1}^{*}, x_{2}^{*}\right)$, which would contradict (B). If $(\mathrm{A})$ did not hold for some $x_{0}>x_{2}^{*}$, then $L\left(x_{1}^{*}, x_{0}\right)>L\left(x_{1}^{*}, x_{2}^{*}\right)$, which would again contradict $(\mathrm{B})$.

Lemma F.1. $J(V)$ is a concave function.

Proof. Let $V_{1}$ and $V_{2}$ be two arbitrary values in $[\underline{x}, \bar{x}]$, and let $V_{\alpha}=\alpha V_{1}+(1-\alpha) V_{2}$, where $\alpha \in(0,1)$. Denote $\left(x_{1 i}^{*}, x_{2 i}^{*}\right)$ as the solution to the maximization problem when $V=V_{i}$, where $i \in\{1,2, \alpha\}$. We show that $J\left(V_{\alpha}\right) \geq \alpha J\left(V_{1}\right)+(1-\alpha) J\left(V_{2}\right)$. 
Applying (A) to any $x \in\left[x_{11}^{*}, x_{21}^{*}\right]$, we know that $K(x)$ cannot lie above the extension of $\overline{x_{1 \alpha}^{*} x_{2 \alpha}^{*}}$. Thus, all points on $\overline{x_{11}^{*} x_{21}^{*}}$ must lie on or below the extension of $\overline{x_{1 \alpha}^{*} x_{2 \alpha}^{*}}$. This implies that $J\left(V_{1}\right) \leq J\left(V_{\alpha}\right)-L_{\alpha}\left(V_{\alpha}-V_{1}\right)$, where $L_{\alpha}=L\left(x_{1 \alpha}^{*}, x_{2 \alpha}^{*}\right)$. Similarly, applying (A) to any $x \in\left[x_{12}^{*}, x_{22}^{*}\right]$ yields: $J\left(V_{2}\right) \leq J\left(V_{\alpha}\right)+L_{\alpha}\left(V_{2}-V_{\alpha}\right)$. Thus,

$$
\alpha J\left(V_{1}\right)+(1-\alpha) J\left(V_{2}\right) \leq J\left(V_{\alpha}\right)+L_{\alpha}\left[\alpha\left(V_{1}-V_{\alpha}\right)+(1-\alpha)\left(V_{2}-V_{\alpha}\right)\right]=J\left(V_{\alpha}\right)
$$

This completes the proof of the lemma. 

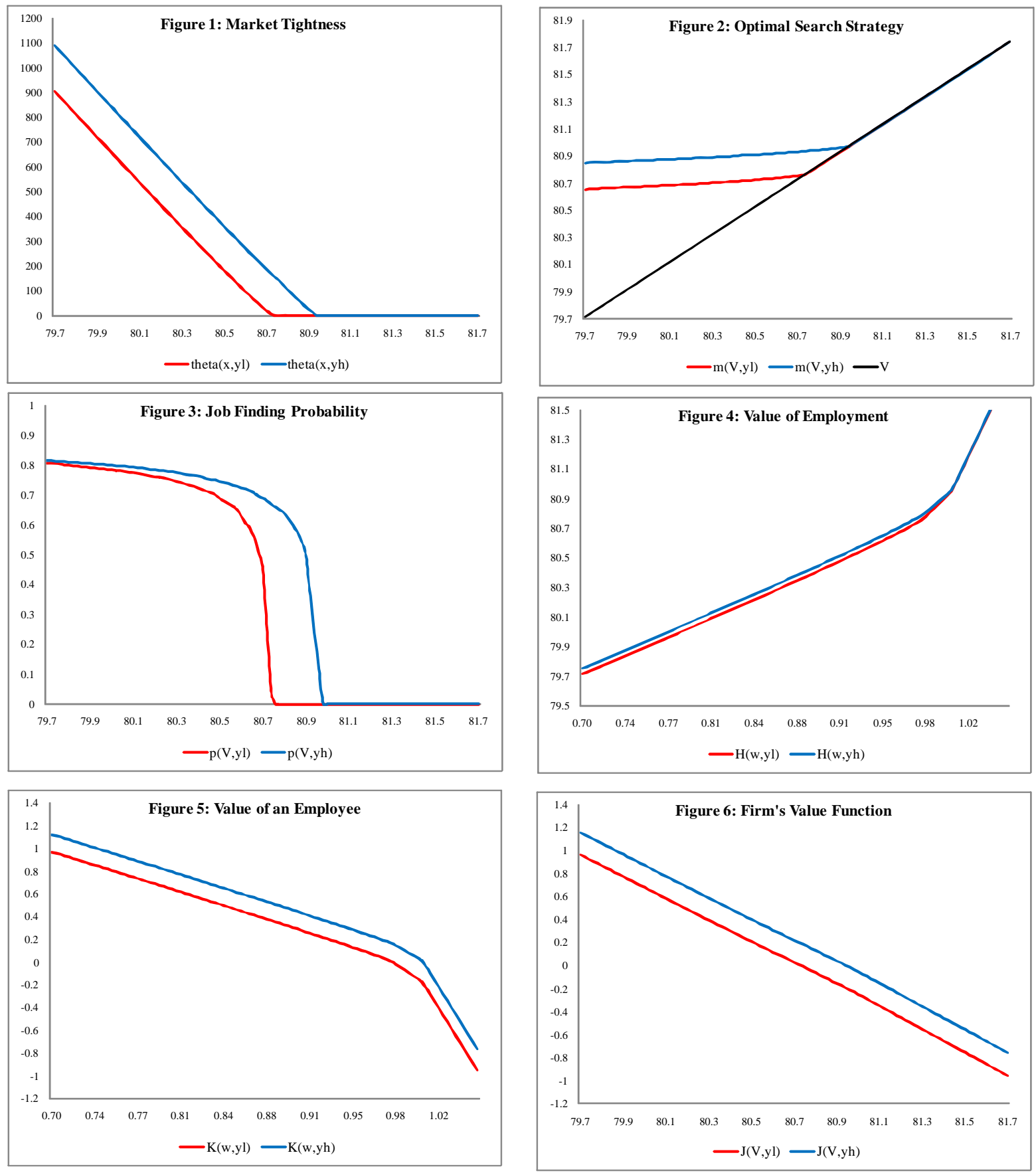